\title{
Comparative quantitative toxicology and QSAR modeling of the haloacetonitriles: forcing agents of water disinfection by-product toxicity
}

\section{Supporting Information}

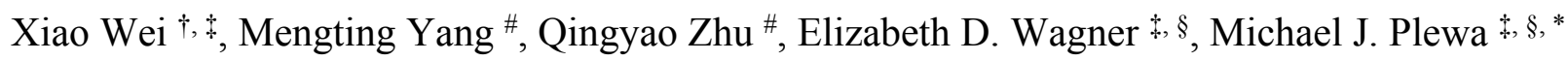

$\uparrow$ Department of Occupational and Environmental Health, School of Public Health, Guangxi Medical University, Nanning, Guangxi 530021, China.

$¥$ Department of Crop Sciences, University of Illinois at Urbana-Champaign, Urbana, IL, 61801, USA

$\S$ Safe Global Water Institute, University of Illinois at Urbana-Champaign, Urbana, IL, 61801, USA

\# College of Chemistry and Environmental Engineering, Shenzhen University, Shenzhen, Guangdong 518000 China

* Corresponding author. Department of Crop Sciences and the Safe Global Water Institute, University of Illinois at Urbana-Champaign, 1101 W. Peabody Dr., Urbana, IL, 61801, USA E-mail address: mplewa@illinois.edu (M.J. Plewa). Co-corresponding author. X. Wei.

Contains 47 pages, 27 tables, and 30 figures 


\section{QSAR model validation}

According to the acceptable criteria reported previously $\left(R^{2}>0.700, Q^{2}{ }_{\text {LOO }}>0.600, p<0.05\right),{ }^{1}$ the obtained $R^{2}, Q^{2}$ LOO and $p$ values indicate the two models with high goodness-of-fit and robustness. The RMSE and $M A E$ values for both models were relatively small. The $F$ values were relatively greater in certain degrees of freedom. The applicability domains which indicated the areas of reliable predictions of the models were characterized using the Williams plot. ${ }^{1}$ The HANs in the two data sets are in the corresponding domains, indicating that both the cytotoxicity and genotoxicity data sets have great representativeness (Figures $4 \mathrm{a}$ and 5a). According to the Yscrambling test criteria, the obtained random models have significantly lower prediction accuracies than the two developed models based on experimental data, indicating no accidental correlation in the QSAR models (Figures $4 \mathrm{~b}$ and $5 \mathrm{~b}) .{ }^{2}$ The variance inflation factor $(V I F)$ of the two variables is lower than 10 , indicating that there is no serious multi-collinearity among the variables and the established models are stable and acceptable. The plot of observed versus predicted $\log \left(\mathrm{LC}_{50}\right)^{-1}$ values is shown in Figure $4 \mathrm{c}$, further demonstrating that the $\mathrm{LC}_{50}$ values predicted from the developed cytotoxicity model are generally coincident with the observed values. Also, the predicted 50\% Tail DNA values generally coincide with the observed values (Figure 5c). 


\begin{tabular}{|c|c|c|c|}
\hline \multicolumn{4}{|c|}{ Table S1. Haloacetonitrile source and purity } \\
\hline $\begin{array}{l}\text { Haloacetonitrile } \\
\text { \& Abbreviation }\end{array}$ & $\begin{array}{l}\text { Formula \& } \\
\text { CAS }\end{array}$ & $\begin{array}{c}\text { Molecular } \\
\text { Weight }\end{array}$ & $\begin{array}{l}\text { Source \& } \\
\text { Purity }\end{array}$ \\
\hline $\begin{array}{l}\text { Iodoacetonitrile } \\
\text { IAN }\end{array}$ & $\begin{array}{l}\mathrm{C}_{2} \mathrm{H}_{2} \mathrm{IN} \\
624-75-9\end{array}$ & 166.95 & $\begin{array}{c}\text { Sigma } \\
\text { Aldrich } \\
98 \%\end{array}$ \\
\hline $\begin{array}{l}\text { Bromoacetonitrile } \\
\text { BAN }\end{array}$ & $\begin{array}{l}\mathrm{C}_{2} \mathrm{H}_{2} \mathrm{BrN} \\
590-17-0\end{array}$ & 119.95 & $\begin{array}{l}\text { Chem } \\
\text { Service } \\
97 \%\end{array}$ \\
\hline $\begin{array}{l}\text { Dibromoacetonitrile } \\
\text { DBAN }\end{array}$ & $\begin{array}{l}\mathrm{C}_{2} \mathrm{HBr}_{2} \mathrm{~N} \\
3252-43-5\end{array}$ & 198.84 & $\begin{array}{l}\text { Chem } \\
\text { Service } \\
97 \%\end{array}$ \\
\hline $\begin{array}{l}\text { Bromochloroacetonitrile } \\
\text { BCAN }\end{array}$ & $\begin{array}{l}\mathrm{C}_{2} \mathrm{HBrClN} \\
83463-62-1\end{array}$ & 154.39 & $\begin{array}{c}\text { Chem } \\
\text { Service } \\
\text { Tech } \\
\end{array}$ \\
\hline $\begin{array}{l}\text { Tribromoacetonitrile } \\
\text { TBAN }\end{array}$ & $\begin{array}{c}\mathrm{C}_{2} \mathrm{Br}_{3} \mathrm{~N} \\
75519-19-6\end{array}$ & 277.74 & $\begin{array}{l}\text { Cansyn Chem } \\
\text { Corp. }>90 \%\end{array}$ \\
\hline $\begin{array}{l}\text { Chloroacetonitrile } \\
\text { CAN }\end{array}$ & $\begin{array}{l}\mathrm{C}_{2} \mathrm{H}_{2} \mathrm{ClN} \\
107-14-2\end{array}$ & 75.497 & $\begin{array}{c}\text { Chem } \\
\text { Service } \\
99.5 \% \\
\end{array}$ \\
\hline $\begin{array}{l}\text { Dichloroacetonitrile } \\
\text { DCAN }\end{array}$ & $\begin{array}{c}\mathrm{C}_{2} \mathrm{HCl}_{2} \mathrm{~N} \\
3018-12-0\end{array}$ & 109.94 & $\begin{array}{l}\text { Chem } \\
\text { Service } \\
99.5 \%\end{array}$ \\
\hline $\begin{array}{l}\text { Trichloroacetonitrile } \\
\text { TCAN }\end{array}$ & $\begin{array}{c}\mathrm{C}_{2} \mathrm{Cl}_{3} \mathrm{~N} \\
545-06-2\end{array}$ & 144.39 & $\begin{array}{c}\text { Sigma } \\
\text { Aldrich } \\
98 \%\end{array}$ \\
\hline $\begin{array}{l}\text { Bromodichloroacetonitrile } \\
\text { BDCAN }\end{array}$ & $\begin{array}{l}\mathrm{C}_{2} \mathrm{BrCl}_{2} \mathrm{~N} \\
60523-73-1\end{array}$ & 188.84 & $\begin{array}{c}\text { Toronto } \\
\text { Res. Chem. 98\% }\end{array}$ \\
\hline $\begin{array}{l}\text { Chlorodibromoacetonitrile } \\
\text { CDBAN }\end{array}$ & $\begin{array}{c}\mathrm{C}_{2} \mathrm{Br}_{2} \mathrm{ClN} \\
144772-39-4\end{array}$ & 233.29 & $\begin{array}{l}\text { Cansyn Chem } \\
\text { Corp. }>88 \%\end{array}$ \\
\hline
\end{tabular}

\section{Methods for statistical analyses for the analytical biology}

Statistical analyses were conducted for each toxicological assay. After a concentration-response curve from combined replicate experiments $(>3)$ was generated, a test for significance using a one-way analysis of variance (ANOVA) test was conducted. If a significant $F$ value of $P \leq 0.05$ was obtained, a Holm-Sidak multiple comparison versus the control group analysis was conducted with the power $(1-\beta) \geq 0.8$ at $\alpha=0.05$ to identify the lowest concentration that was 
significantly different from the negative control. ${ }^{3,4}$ After regression analyses, $\mathrm{LC}_{50}$ values were determined for $\mathrm{CHO}$ cell cytotoxicity, 50\% Tail DNA values for $\mathrm{CHO}$ cell genotoxicity, and $\mathrm{EC}_{50}$ values for NAC-thiol reactivity. Bootstrap statistics were conducted for each assay dataset 5,6 and mean toxicity index values $( \pm \mathrm{SE})$ were calculated. We used index values (expressed as $\mu \mathrm{M})$ such that the larger the value, the more toxic or reactive the sample. The cytotoxicity index (CTI) value is the $\mathrm{LC}_{50}{ }^{-1} \times 10^{3}$; the genotoxicity index (GTI) value is the $50 \%$ Tail $\mathrm{DNA}^{-1} \times 10^{3}$; the thiol reactivity index (TRI) value is the $\mathrm{EC}_{50}{ }^{-1} \times 10^{3}$. Using these index values, an ANOVA test was conducted to identify significant differences among specific groups. The Pearson product-moment correlation test analyzed functional associations amongst HAN groups and biological and physicochemical metrics. ${ }^{7,8}$

\section{Biological and chemical reagents, $\mathrm{CHO}$ cells}

For the in vitro cytotoxicity and genotoxicity experiments CHO K1 cell line (AS52, clone 11-48) was employed. ${ }^{9,}{ }^{10}$ Cells were grown in Hams F12 medium containing 5\% fetal bovine serum (FBS), $1 \%$ L-glutamine, and $1 \%$ antibiotics $(0.25 \mu \mathrm{g} / \mathrm{mL}$ amphotericin B, $100 \mu \mathrm{g} / \mathrm{mL}$ streptomycin sulfate, and 100 units $/ \mathrm{mL}$ sodium penicillin $\mathrm{G}$ in $0.85 \%$ saline) at $37^{\circ} \mathrm{C}$ in a mammalian cell incubator with a humidified atmosphere of $5 \% \mathrm{CO}_{2}$.

\section{CHO cell chronic cytotoxicity analyses}

$\mathrm{CHO}$ cell cytotoxicity was measured as the reduction in cell density after exposure of $\mathrm{CHO}$ cells to each HAN for $72 \mathrm{~h}$ compared to untreated concurrent negative controls. ${ }^{11}$ Cytotoxicity uncovers a wide array of toxic insults and adverse biological impacts. In this study the 
cytotoxicity of TBAN, BDCAN and CDBAN was analyzed; data for the other HANs were previously published. ${ }^{12}$ Detailed procedures for this assay were published. ${ }^{11,13}$

The concentration-response graphs illustrating the $\mathrm{CHO}$ cell chronic cytotoxicity of the haloacetonitriles (HANs) are presented in Figures S1 to S10. 


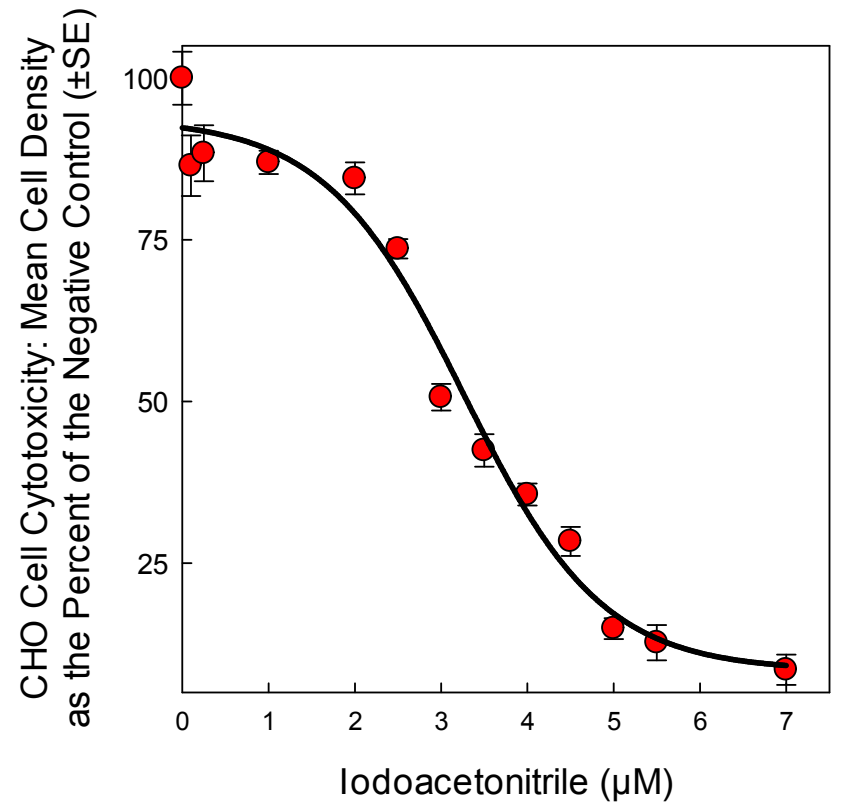

Figure S1. CHO cell cytotoxicity concentration-response curve for IAN. Mean $\left( \pm\right.$ SE) $\mathrm{LC}_{50}$ value was $3.27 \pm 0.05 \mu \mathrm{M}$.

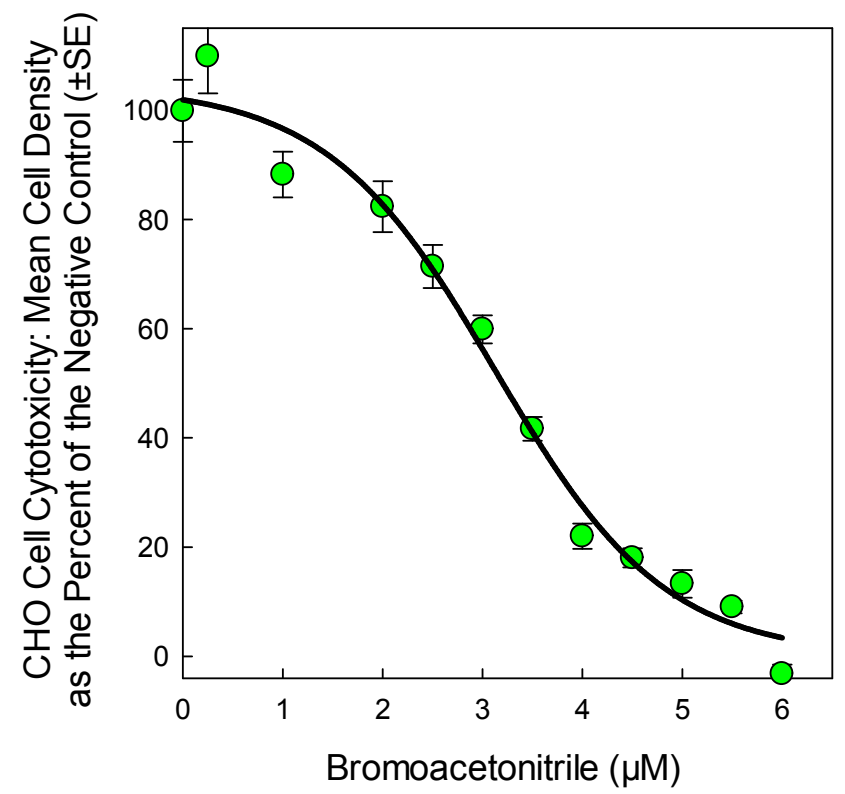

Figure S2. CHO cell cytotoxicity concentration-response curve for BAN. Mean $( \pm \mathrm{SE}) \mathrm{LC}_{50}$ value was $3.10 \pm 0.06 \mu \mathrm{M}$. 

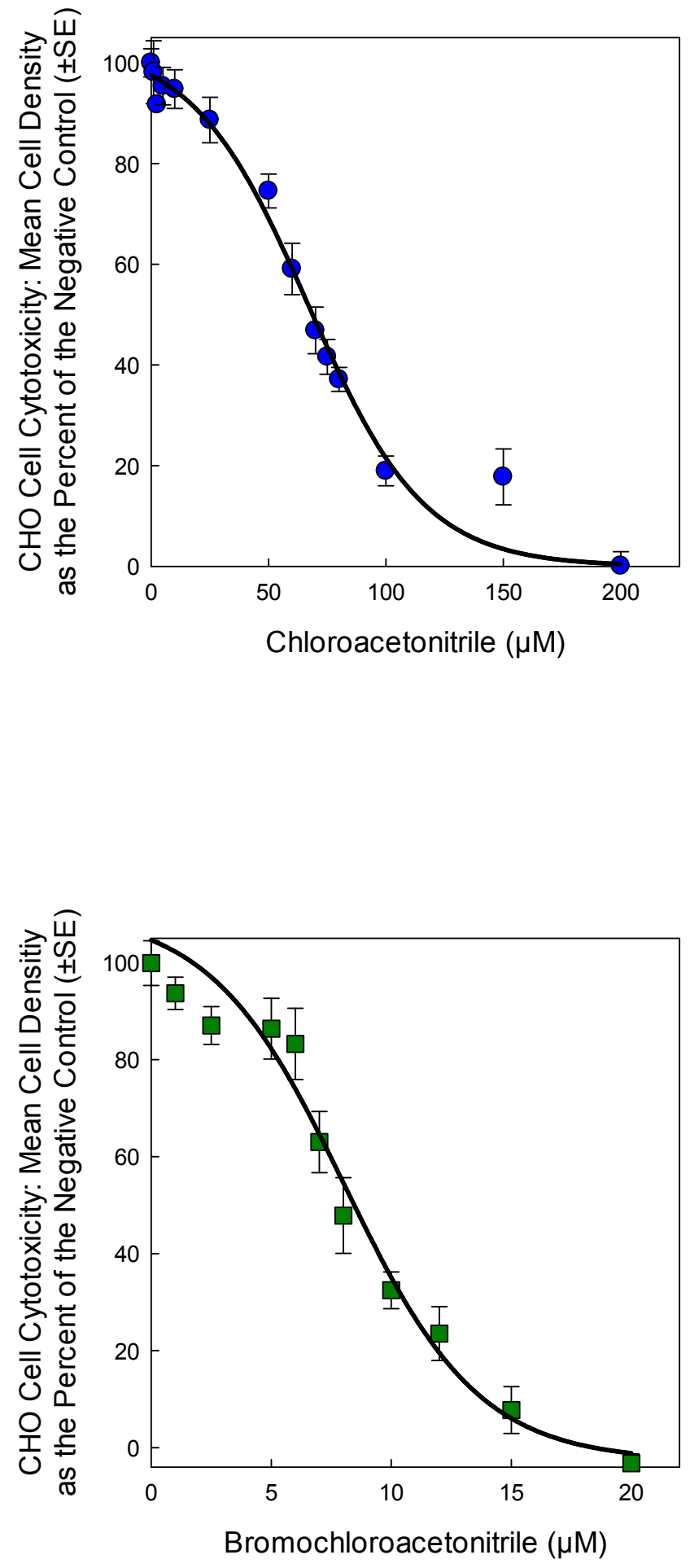

Figure S3. CHO cell cytotoxicity concentration-response curve for CAN. Mean $( \pm \mathrm{SE}) \mathrm{LC}_{50}$ value was $66.09 \pm 1.63 \mu \mathrm{M}$.
Figure S4. CHO cell cytotoxicity concentration-response curve for BCAN. Mean $\left( \pm\right.$ SE) LC $_{50}$ value was $8.20 \pm 0.51 \mu \mathrm{M}$. 


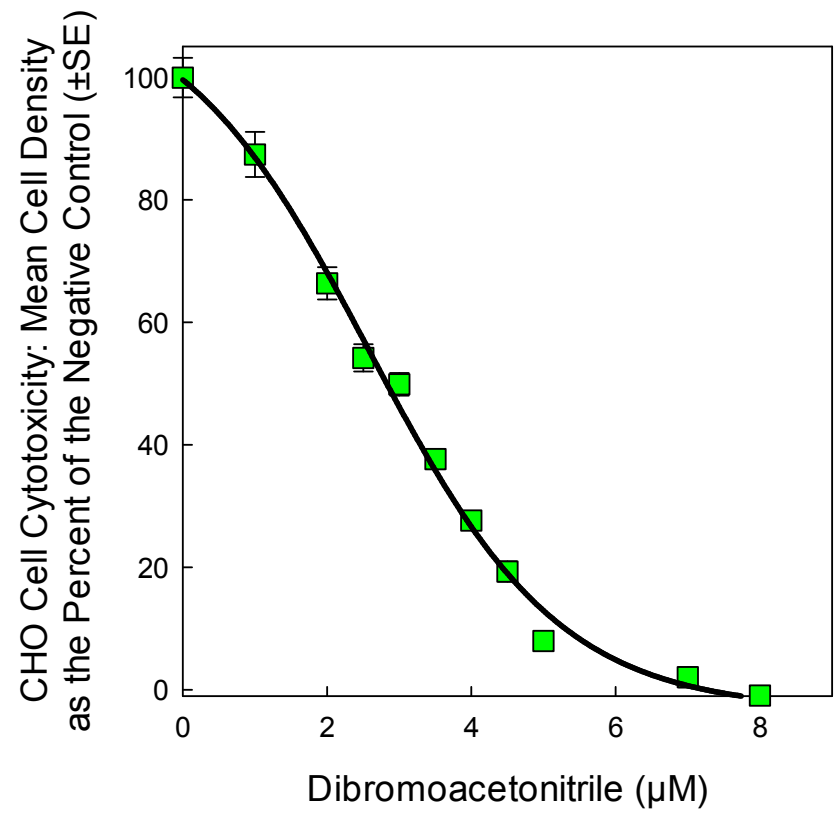

Figure S5. CHO cell cytotoxicity concentration-response curve for DBAN. Mean $( \pm \mathrm{SE}) \mathrm{LC}_{50}$ value was $2.79 \pm 0.09 \mu \mathrm{M}$.

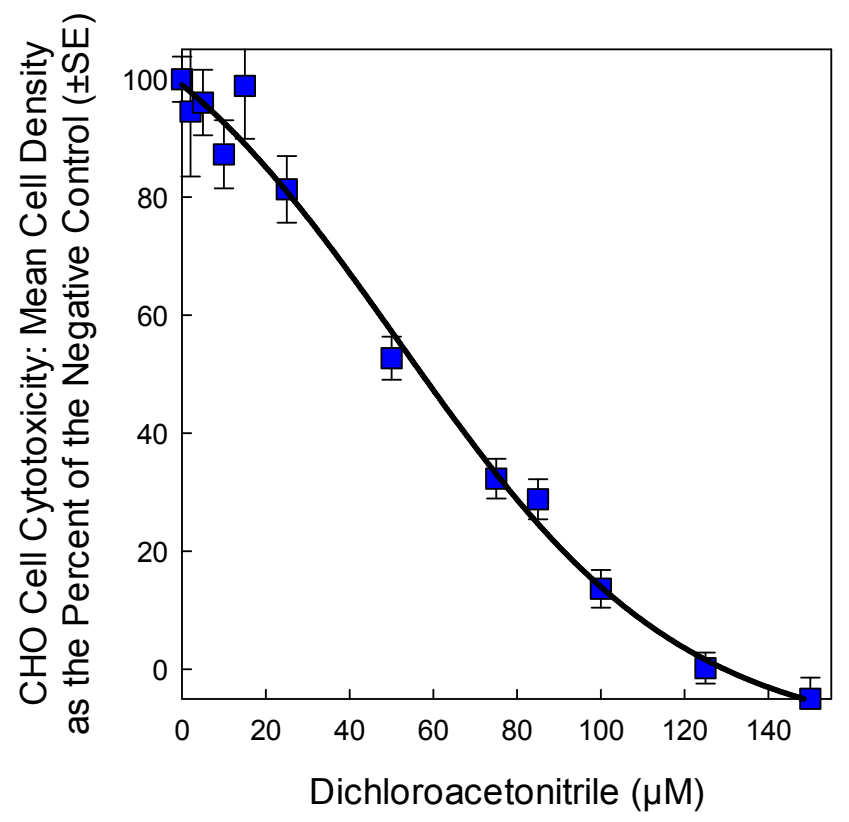

Figure S6. CHO cell cytotoxicity concentration-response curve for DCAN. Mean $( \pm \mathrm{SE}) \mathrm{LC}_{50}$ value was $55.03 \pm 3.23 \mu \mathrm{M}$. 

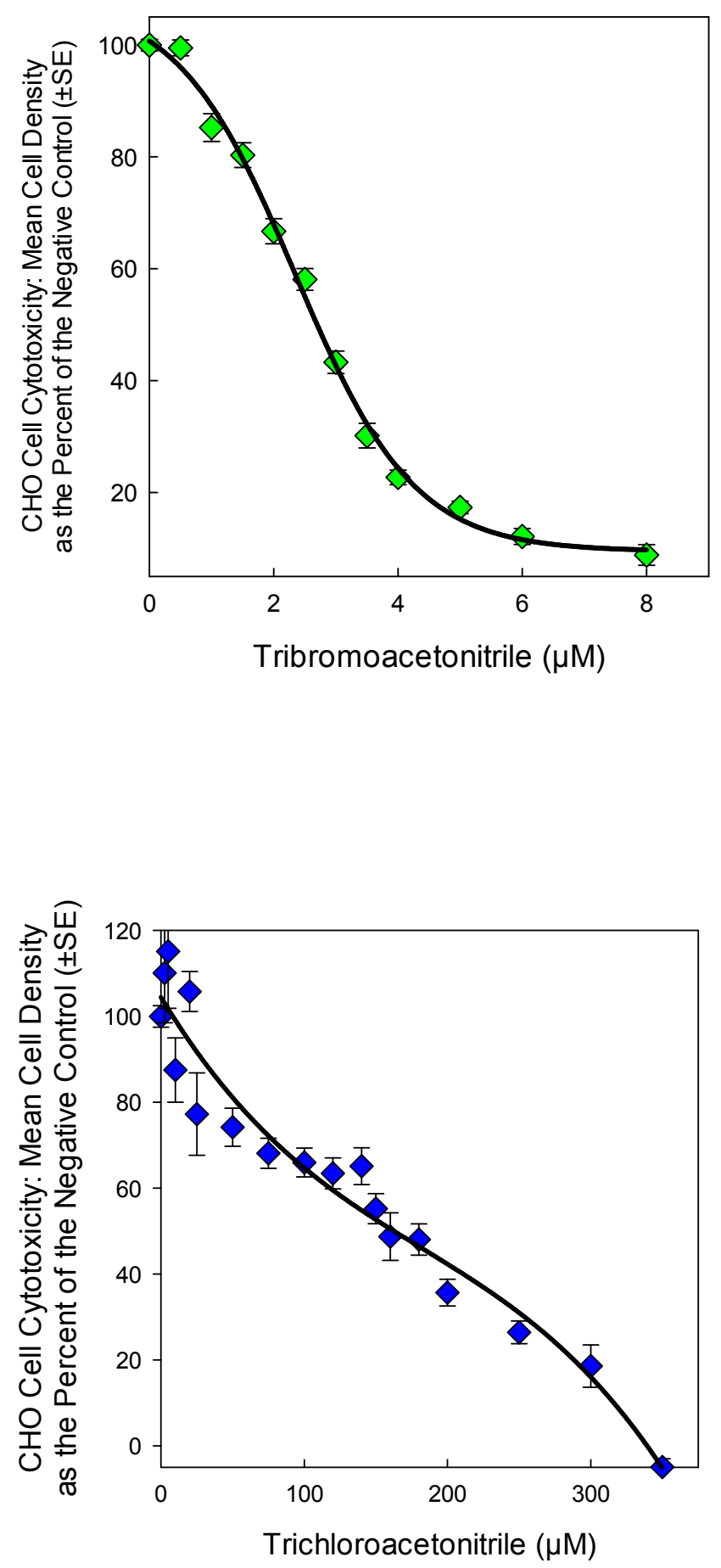

Figure S7. CHO cell cytotoxicity concentration-response curve for TBAN. Mean $( \pm \mathrm{SE}) \mathrm{LC}_{50}$ value was $2.71 \pm 0.04 \mu \mathrm{M}$.
Figure S8. CHO cell cytotoxicity concentration-response curve for TCAN. Mean $\left( \pm\right.$ SE) $\mathrm{LC}_{50}$ value was $158.55 \pm 6.01 \mu \mathrm{M}$. 

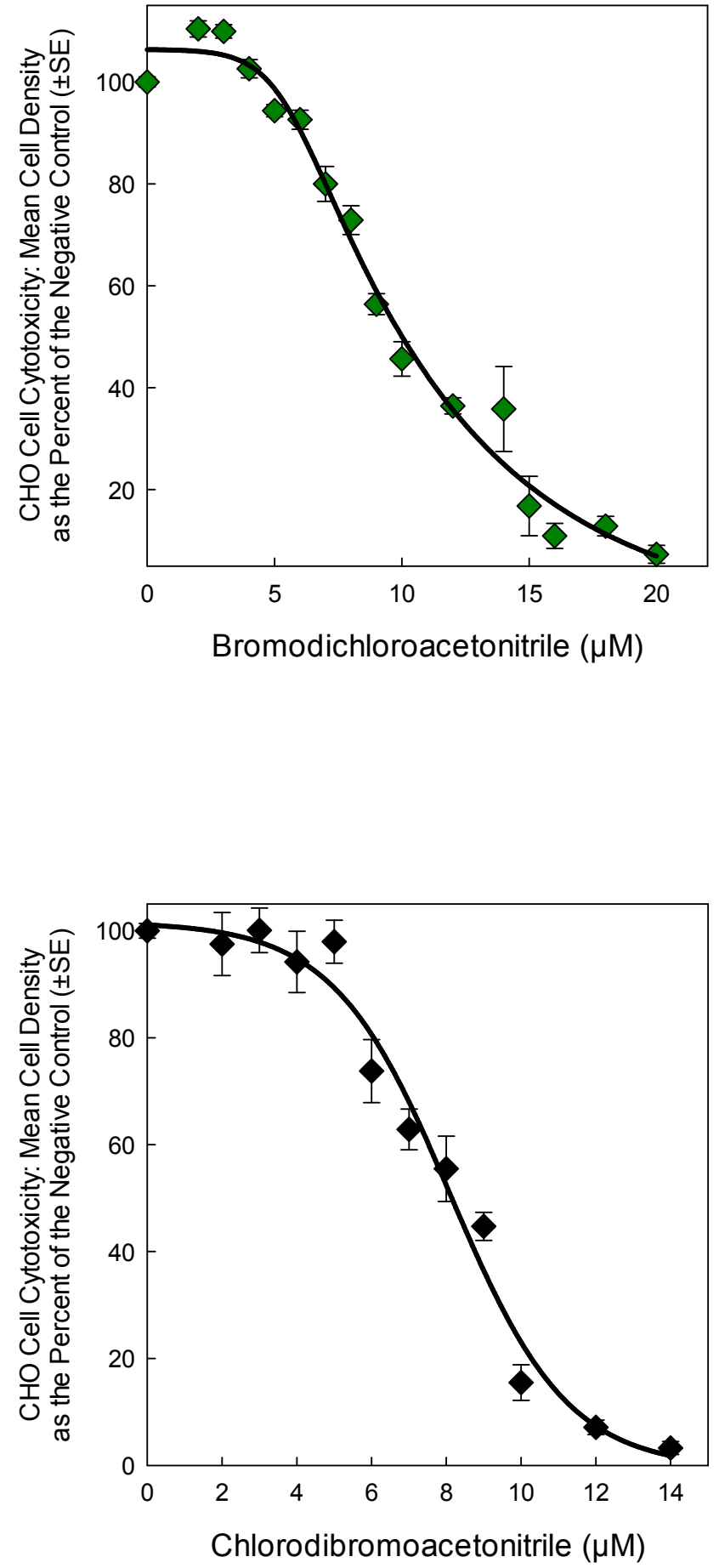

Figure S9. CHO cell cytotoxicity concentration-response curve for BDCAN. Mean $( \pm \mathrm{SE}) \mathrm{LC}_{50}$ value was $10.22 \pm 0.12 \mu \mathrm{M}$.
Figure S10. CHO cell cytotoxicity concentration-response curve for CDBAN. Mean $( \pm \mathrm{SE}) \mathrm{LC}_{50}$ value was $8.14 \pm 0.18 \mu \mathrm{M}$. 
The ANOVA test statistic to determine significant decreases in cell viability for the three newly evaluated HANs are presented in Table S2 (TBAN), Table S3 (BDCAN) and Table S4

(CDBAN).

Table S2. One Way Analysis of Variance: Tribromoacetonitrile (TBAN) CHO cell cytotoxicity. Percent of the negative control.

\begin{tabular}{|c|c|c|c|c|c|c|c|}
\hline Group Name & $\mathbf{N}$ & Missing & Mean & Std Dev & SEM & & \\
\hline 0 TBAN $\mu \mathrm{M}$ & 24 & 0 & 99.974 & 5.116 & 1.044 & & \\
\hline 0.5 TBAN $\mu \mathrm{M}$ & 4 & 0 & 99.478 & 2.826 & 1.413 & & \\
\hline $1 \mathrm{TBAN} \mu \mathrm{M}$ & 8 & 0 & 85.243 & 7.056 & 2.495 & & \\
\hline 1.5 TBAN $\mu \mathrm{M}$ & 8 & 0 & 80.312 & 6.252 & 2.210 & & \\
\hline 2 TBAN $\mu \mathrm{M}$ & 10 & 0 & 66.713 & 7.067 & 2.235 & & \\
\hline 2.5 TBAN $\mu \mathrm{M}$ & 8 & 0 & 58.093 & 5.508 & 1.948 & & \\
\hline 3 TBAN $\mu \mathrm{M}$ & 8 & 0 & 43.266 & 5.667 & 2.003 & & \\
\hline 3.5 TBAN $\mu \mathrm{M}$ & 8 & 0 & 30.159 & 6.233 & 2.204 & & \\
\hline 4 TBAN $\mu \mathrm{M}$ & 10 & 0 & 22.686 & 4.133 & 1.307 & & \\
\hline 5 TBAN $\mu \mathrm{M}$ & 8 & 0 & 17.336 & 3.137 & 1.109 & & \\
\hline 6 TBAN $\mu \mathrm{M}$ & 10 & 0 & 12.131 & 4.548 & 1.438 & & \\
\hline $8 \mathrm{TBAN} \mu \mathrm{M}$ & 6 & 0 & 8.824 & 4.506 & 1.840 & & \\
\hline \multicolumn{2}{|c|}{ Source of Variation } & DF & SS & \multicolumn{2}{|c|}{ MS } & $\mathbf{F}$ & $\mathbf{P}$ \\
\hline \multicolumn{2}{|c|}{ Between Groups } & 11 & 128912.588 & \multicolumn{2}{|c|}{11719.326} & 401.784 & $<0.001$ \\
\hline \multicolumn{2}{|c|}{ Residual } & 100 & 2916.820 & \multicolumn{2}{|c|}{29.168} & & \\
\hline \multicolumn{2}{|l|}{ Total } & 111 & 131829.409 & & & & \\
\hline
\end{tabular}

The differences in the mean values among the treatment groups are greater than would be expected by chance; there is a statistically significant difference $\mathrm{P}=<0.001$ ).

Power of performed test with alpha $=0.050: 1.000$

Multiple Comparisons versus Control Group (Holm-Sidak method):

Overall significance level $=0.05$

Comparisons for factor:

\begin{tabular}{|c|c|c|c|c|}
\hline Comparison & Diff of Means & $\mathbf{t}$ & $\mathbf{P}$ & $\mathbf{P}<\mathbf{0 . 0 5 0}$ \\
\hline 0 TBAN $\mu \mathrm{M}$ vs. 6 TBAN $\mu \mathrm{M}$ & 87.843 & 43.213 & $<0.001$ & Yes \\
\hline 0 TBAN $\mu \mathrm{M}$ vs. 4 TBAN $\mu \mathrm{M}$ & 77.288 & 38.021 & $<0.001$ & Yes \\
\hline 0 TBAN $\mu \mathrm{M}$ vs. 5 TBAN $\mu \mathrm{M}$ & 82.639 & 37.480 & $<0.001$ & Yes \\
\hline 0 TBAN $\mu \mathrm{M}$ vs. 8 TBAN $\mu \mathrm{M}$ & 91.150 & 36.976 & $<0.001$ & Yes \\
\hline 0 TBAN $\mu \mathrm{M}$ vs. 3.5 TBAN $\mu \mathrm{M}$ & 69.815 & 31.664 & $<0.001$ & Yes \\
\hline 0 TBAN $\mu$ v vs. 3 TBAN $\mu \mathrm{M}$ & 56.709 & 25.720 & $<0.001$ & Yes \\
\hline 0 TBAN $\mu \mathrm{M}$ vs. 2.5 TBAN $\mu \mathrm{M}$ & 41.881 & 18.995 & $<0.001$ & Yes \\
\hline 0 TBAN $\mu \mathrm{M}$ vs. 2 TBAN $\mu \mathrm{M}$ & 33.261 & 16.363 & $<0.001$ & Yes \\
\hline 0 TBAN $\mu \mathrm{M}$ vs. 1.5 TBAN $\mu \mathrm{M}$ & 19.662 & 8.918 & $<0.001$ & Yes \\
\hline 0 TBAN $\mu \mathrm{M}$ vs. 1 TBAN $\mu \mathrm{M}$ & 14.731 & 6.681 & $<0.001$ & Yes \\
\hline 0 TBAN $\mu \mathrm{M}$ vs. 0.5 TBAN $\mu \mathrm{M}$ & 0.497 & 0.170 & 0.865 & No \\
\hline
\end{tabular}


Table S3. One Way Analysis of Variance: Bromodichloroacetonitrile (BDCAN) CHO cell cytotoxicity. Percent of the negative control.

\begin{tabular}{|c|c|c|c|c|c|c|c|}
\hline Group Name & $\mathbf{N}$ & Missing & Mean & Std Dev & \multicolumn{3}{|c|}{ SEM } \\
\hline 0 BDCAN & 32 & 0 & 100.007 & 5.657 & \multicolumn{3}{|c|}{1.000} \\
\hline 2 BDCAN & 9 & 0 & 110.412 & 4.775 & \multicolumn{3}{|c|}{1.592} \\
\hline 3 BDCAN & 4 & 0 & 109.911 & 2.635 & \multicolumn{3}{|c|}{1.318} \\
\hline 4 BDCAN & 13 & 0 & 102.589 & 6.452 & \multicolumn{3}{|c|}{1.790} \\
\hline 5 BDCAN & 8 & 0 & 94.370 & 3.412 & \multicolumn{3}{|c|}{1.206} \\
\hline 6 BDCAN & 17 & 0 & 92.592 & 7.649 & \multicolumn{3}{|c|}{1.855} \\
\hline 7 BDCAN & 4 & 0 & 79.994 & 6.856 & \multicolumn{3}{|c|}{3.428} \\
\hline 8 BDCAN & 13 & 0 & 72.903 & 10.203 & \multicolumn{3}{|c|}{2.830} \\
\hline 9 BDCAN & 4 & 0 & 56.405 & 4.114 & \multicolumn{3}{|c|}{2.057} \\
\hline 10 BDCAN & 9 & 0 & 45.637 & 10.176 & \multicolumn{3}{|c|}{3.392} \\
\hline 12 BDCAN & 8 & 0 & 36.424 & 4.518 & \multicolumn{3}{|c|}{1.597} \\
\hline 14 BDCAN & 8 & 0 & 35.808 & 23.578 & \multicolumn{3}{|c|}{8.336} \\
\hline 15 BDCAN & 5 & 0 & 16.787 & 13.050 & \multicolumn{3}{|c|}{5.836} \\
\hline 16 BDCAN & 4 & 0 & 10.924 & 4.901 & \multicolumn{3}{|c|}{2.451} \\
\hline 18 BDCAN & 4 & 0 & 12.848 & 3.847 & \multicolumn{3}{|c|}{1.924} \\
\hline 20 BDCAN & 5 & 0 & 7.322 & 3.932 & \multicolumn{3}{|c|}{1.758} \\
\hline \multicolumn{2}{|c|}{ Source of Variation } & DF & SS & \multicolumn{2}{|c|}{ MS } & F & $\mathbf{P}$ \\
\hline \multicolumn{2}{|c|}{ Between Groups } & 15 & 159535.937 & \multicolumn{2}{|c|}{10635.729} & 141.080 & $<0.001$ \\
\hline Residual & & 131 & 9875.827 & \multicolumn{2}{|c|}{75.388} & & \\
\hline Total & & 146 & 169411.764 & & & & \\
\hline
\end{tabular}

The differences in the mean values among the treatment groups are greater than would be expected by chance; there is a statistically significant difference $(\mathrm{P}=<0.001)$.

Power of performed test with alpha $=0.050: 1.000$

Multiple Comparisons versus Control Group (Holm-Sidak method):

Overall significance level $=0.05$

Comparisons for factor:

$\begin{array}{lcccc}\text { Comparison } & \text { Diff of Means } & \mathbf{t} & \mathbf{P} & \mathbf{P}<\mathbf{0 . 0 5 0} \\ \text { 0 BDCAN vs. 20 BDCAN } & 92.685 & 22.198 & <0.001 & \text { Yes } \\ \text { 0 BDCAN vs. 15 BDCAN } & 83.220 & 19.931 & <0.001 & \text { Yes } \\ \text { 0 BDCAN vs. 16 BDCAN } & 89.083 & 19.346 & <0.001 & \text { Yes } \\ \text { 0 BDCAN vs. 18 BDCAN } & 87.159 & 18.928 & <0.001 & \text { Yes } \\ \text { 0 BDCAN vs. 14 BDCAN } & 64.200 & 18.706 & <0.001 & \text { Yes } \\ \text { 0 BDCAN vs. 12 BDCAN } & 63.583 & 18.526 & <0.001 & \text { Yes } \\ \text { 0 BDCAN vs. 10 BDCAN } & 54.370 & 16.596 & <0.001 & \text { Yes } \\ \text { 0 BDCAN vs. 8 BDCAN } & 27.104 & 9.491 & <0.001 & \text { Yes } \\ \text { 0 BDCAN vs. 9 BDCAN } & 43.602 & 9.469 & <0.001 & \text { Yes } \\ \text { 0 BDCAN vs. 7 BDCAN } & 20.014 & 4.346 & <0.001 & \text { Yes } \\ \text { 0 BDCAN vs. 2 BDCAN } & 10.405 & 3.176 & 0.009 & \text { Yes } \\ \text { 0 BDCAN vs. 6 BDCAN } & 7.415 & 2.846 & 0.020 & \text { Yes } \\ \text { 0 BDCAN vs. 3 BDCAN } & 9.904 & 2.151 & 0.097 & \text { No } \\ \text { 0 BDCAN vs. 5 BDCAN } & 5.637 & 1.642 & 0.195 & \text { No } \\ \text { 0 BDCAN vs. 4 BDCAN } & 2.582 & 0.904 & 0.368 & \text { No }\end{array}$


Table S4. One Way Analysis of Variance: Chlorodibromoacetonitrile (CDBAN) CHO cell cytotoxicity. Percent of the negative control.

\begin{tabular}{|c|c|c|c|c|c|c|c|}
\hline Group Name & $\mathbf{N}$ & Missing & Mean & Std Dev & \multicolumn{3}{|c|}{ SEM } \\
\hline OuM CDBAN & 16 & 0 & 100.007 & 5.512 & \multicolumn{3}{|c|}{1.378} \\
\hline 2 CDBAN & 5 & 0 & 97.517 & 13.185 & \multicolumn{3}{|c|}{5.897} \\
\hline 3 CDBAN & 4 & 0 & 100.095 & 8.360 & \multicolumn{3}{|c|}{4.180} \\
\hline 4 CDBAN & 9 & 0 & 94.191 & 17.199 & \multicolumn{3}{|c|}{5.733} \\
\hline 5 CDBAN & 8 & 0 & 97.966 & 11.399 & \multicolumn{3}{|c|}{4.030} \\
\hline 6 CDBAN & 9 & 0 & 73.771 & 17.656 & \multicolumn{3}{|c|}{5.885} \\
\hline 7 CDBAN & 4 & 0 & 62.866 & 7.620 & \multicolumn{3}{|c|}{3.810} \\
\hline 8 CDBAN & 9 & 0 & 55.506 & 18.335 & \multicolumn{3}{|c|}{6.112} \\
\hline 9 CDBAN & 4 & 0 & 44.707 & 5.272 & \multicolumn{3}{|c|}{2.636} \\
\hline $10 \mathrm{CDBAN}$ & 9 & 0 & 15.487 & 9.994 & \multicolumn{3}{|c|}{3.331} \\
\hline 12 CDBAN & 9 & 0 & 7.124 & 4.014 & \multicolumn{3}{|c|}{1.338} \\
\hline 14 CDBAN & 5 & 0 & 3.261 & 2.716 & \multicolumn{3}{|c|}{1.215} \\
\hline \multicolumn{2}{|c|}{ Source of Variation } & DF & SS & \multicolumn{2}{|c|}{ MS } & $\mathbf{F}$ & $\mathbf{P}$ \\
\hline \multicolumn{2}{|c|}{ Between Groups } & 11 & 120619.108 & \multirow{2}{*}{\multicolumn{2}{|c|}{$\begin{array}{r}10965.373 \\
139688\end{array}$}} & 78.499 & $<0.001$ \\
\hline \multicolumn{2}{|c|}{ Residual } & 79 & 11035.320 & & & & \\
\hline Total & & 90 & 131654.428 & & & \\
\hline
\end{tabular}

The differences in the mean values among the treatment groups are greater than would be expected by chance; there is a statistically significant difference $(\mathrm{P}=<0.001)$.

Power of performed test with alpha $=0.050: 1.000$

Multiple Comparisons versus Control Group (Holm-Sidak method):

Overall significance level $=0.05$

Comparisons for factor:

\section{Comparison}

0 CDBAN vs. 12 CDBAN

0 CDBAN vs. 10 CDBAN

0 CDBAN vs. 14 CDBAN

0 CDBAN vs. 8 CDBAN

0 CDBAN vs. 9 CDBAN

0 CDBAN vs. 7 CDBAN

0 CDBAN vs. 6 CDBAN

0 CDBAN vs. 4 CDBAN

0 CDBAN vs. 2 CDBAN

0 CDBAN vs. 5 CDBAN

0 CDBAN vs. 3 CDBAN

Diff of Means
92.883
84.521
96.746
44.502
55.301
37.141
26.236
5.817
2.490
2.042
0.0874

$\begin{array}{ccc}\mathbf{t} & \mathbf{P} & \mathbf{P}<\mathbf{0 . 0 5 0} \\ 18.861 & <0.001 & \text { Yes } \\ 17.163 & <0.001 & \text { Yes } \\ 15.977 & <0.001 & \text { Yes } \\ 9.037 & <0.001 & \text { Yes } \\ 8.370 & <0.001 & \text { Yes } \\ 5.621 & <0.001 & \text { Yes } \\ 5.328 & <0.001 & \text { Yes } \\ 1.181 & 0.668 & \text { No } \\ 0.411 & 0.968 & \text { No } \\ 0.399 & 0.905 & \text { No } \\ 0.0132 & 0.989 & \text { No }\end{array}$


Table S5. One Way Analysis of Variance: 10 HAN CTI Comparisons.

\begin{tabular}{|c|c|c|c|c|c|c|c|}
\hline Group Name & $\mathbf{N}$ & Missing & Mean & Std Dev & SEM & & \\
\hline IAN CTI & 17 & 0 & 307.280 & 18.180 & 4.40 & & \\
\hline BAN CTI & 25 & 0 & 325.828 & 35.268 & 7.05 & & \\
\hline DBAN CTI & 16 & 0 & 364.565 & 47.938 & 11.98 & & \\
\hline BCAN CTI & 19 & 0 & 130.839 & 35.932 & 8.24 & & \\
\hline TBAN CTI & 14 & 0 & 369.561 & 20.086 & 5.36 & & \\
\hline CAN CTI & 19 & 0 & 15.298 & 1.659 & 0.38 & & \\
\hline DCAN CTI & 19 & 0 & 19.483 & 5.614 & 1.28 & & \\
\hline TCAN CTI & 27 & 0 & 6.546 & 1.293 & 0.24 & & \\
\hline BDCAN CTI & 18 & 0 & 98.097 & 4.737 & 1.11 & & \\
\hline CDBAN CTI & 11 & 0 & 123.593 & 10.668 & 3.21 & & \\
\hline \multicolumn{2}{|c|}{ Source of Variation } & DF & SS & \multicolumn{2}{|c|}{ MS } & $\mathbf{F}$ & $\mathbf{P}$ \\
\hline \multicolumn{2}{|c|}{ Between Groups } & 9 & 3840984.828 & \multicolumn{2}{|c|}{426776.092} & 744.800 & $<0.001$ \\
\hline \multicolumn{2}{|c|}{ Residual } & 175 & 100276.348 & \multicolumn{2}{|c|}{573.008} & & \\
\hline Total & & 184 & 3941261.176 & & & & \\
\hline
\end{tabular}

The differences in the mean values among the treatment groups are greater than would be expected by chance; there is a statistically significant difference $(\mathrm{P}=<0.001)$.

Power of performed test with alpha $=0.050: 1.000$

All Pairwise Multiple Comparison Procedures (Holm-Sidak method): Overall significance level $=0.05$

Comparisons for factor:

Comparison

BAN CTI vs. TCAN CTI

DBAN CTI vs. TCAN CTI

TBAN CTI vs. TCAN CTI

DBAN CTI vs. CAN CTI

BAN CTI vs. CAN CTI

DBAN CTI vs. DCAN CTI

BAN CTI vs. DCAN CTI

TBAN CTI vs. CAN CTI

TBAN CTI vs. DCAN CTI

IAN CTI vs. TCAN CTI

IAN CTI vs. CAN CTI

IAN CTI vs. DCAN CTI

DBAN CTI vs. BDCAN CTI

TBAN CTI vs. BDCAN CTI

BAN CTI vs. BDCAN CTI

DBAN CTI vs. BCAN CTI

TBAN CTI vs. BCAN CTI

BAN CTI vs. BCAN CTI

IAN CTI vs. BDCAN CTI

DBAN CTI vs. CDBAN CTI

TBAN CTI vs. CDBAN CTI

BAN CTI vs. CDBAN CTI

IAN CTI vs. BCAN CTI

IAN CTI vs. CDBAN CTI

BCAN CTI vs. TCAN CTI

BCAN CTI vs. CAN CTI

Diff of Means
319.282
358.019
363.014
349.267
310.530
345.082
306.345
354.262
350.077
300.734
291.982
287.797
266.469
271.464
227.731
233.727
238.722
194.989
209.184
240.973
245.968
202.235
176.441
183.687
124.293
115.541

$\begin{array}{ccc}\mathbf{t} & \mathbf{P} & \mathbf{P}<\mathbf{0 . 0 5 0} \\ 48.056 & <0.001 & \text { Yes } \\ 47.406 & <0.001 & \text { Yes } \\ 46.047 & <0.001 & \text { Yes } \\ 43.001 & <0.001 & \text { Yes } \\ 42.623 & <0.001 & \text { Yes } \\ 42.486 & <0.001 & \text { Yes } \\ 42.048 & <0.001 & \text { Yes } \\ 42.017 & <0.001 & \text { Yes } \\ 41.521 & <0.001 & \text { Yes } \\ 40.577 & <0.001 & \text { Yes } \\ 36.536 & <0.001 & \text { Yes } \\ 36.013 & <0.001 & \text { Yes } \\ 32.398 & <0.001 & \text { Yes } \\ 31.824 & <0.001 & \text { Yes } \\ 30.776 & <0.001 & \text { Yes } \\ 28.776 & <0.001 & \text { Yes } \\ 28.314 & <0.001 & \text { Yes } \\ 26.764 & <0.001 & \text { Yes } \\ 25.839 & <0.001 & \text { Yes } \\ 25.702 & <0.001 & \text { Yes } \\ 25.503 & <0.001 & \text { Yes } \\ 23.350 & <0.001 & \text { Yes } \\ 22.079 & <0.001 & \text { Yes } \\ 19.831 & <0.001 & \text { Yes } \\ 17.340 & <0.001 & \text { Yes } \\ 14.877 & <0.001 & \text { Yes }\end{array}$




$\begin{array}{lrrrr}\text { BCAN CTI vs. DCAN CTI } & 111.355 & 14.338 & <0.001 & \text { Yes } \\ \text { CDBAN CTI vs. TCAN CTI } & 117.047 & 13.670 & <0.001 & \text { Yes } \\ \text { BDCAN CTI vs. TCAN CTI } & 91.550 & 12.569 & <0.001 & \text { Yes } \\ \text { CDBAN CTI vs. CAN CTI } & 108.295 & 11.941 & <0.001 & \text { Yes } \\ \text { CDBAN CTI vs. DCAN CTI } & 104.109 & 11.479 & <0.001 & \text { Yes } \\ \text { BDCAN CTI vs. CAN CTI } & 82.798 & 10.516 & <0.001 & \text { Yes } \\ \text { BDCAN CTI vs. DCAN CTI } & 78.613 & 9.985 & <0.001 & \text { Yes } \\ \text { TBAN CTI vs. IAN CTI } & 62.280 & 7.209 & <0.001 & \text { Yes } \\ \text { DBAN CTI vs. IAN CTI } & 57.285 & 6.871 & <0.001 & \text { Yes } \\ \text { TBAN CTI vs. BAN CTI } & 43.733 & 5.473 & <0.001 & \text { Yes } \\ \text { DBAN CTI vs. BAN CTI } & 38.738 & 5.055 & <0.001 & \text { Yes } \\ \text { BCAN CTI vs. BDCAN CTI } & 32.742 & 4.159 & <0.001 & \text { Yes } \\ \text { CDBAN CTI vs. BDCAN CTI } & 25.496 & 2.783 & 0.041 & \text { Yes } \\ \text { BAN CTI vs. IAN CTI } & 18.548 & 2.465 & 0.085 & \text { No } \\ \text { DCAN CTI vs. TCAN CTI } & 12.937 & 1.805 & 0.315 & \text { No } \\ \text { CAN CTI vs. TCAN CTI } & 8.752 & 1.221 & 0.637 & \text { No } \\ \text { BCAN CTI vs. CDBAN CTI } & 7.246 & 0.799 & 0.810 & \text { No } \\ \text { TBAN CTI vs. DBAN CTI } & 4.995 & 0.570 & 0.814 & \text { No } \\ \text { DCAN CTI vs. CAN CTI } & 4.185 & 0.539 & 0.591 & \text { No }\end{array}$

Single cell gel electrophoresis genotoxicity analyses. Single cell gel electrophoresis (SCGE or comet) quantitatively measures genomic DNA damage including DNA strand breaks, alkalilabile sites, incomplete excision repair sites, and interstrand crosslinks in the nuclei of cells. ${ }^{14-16}$ $\mathrm{CHO}$ cells were treated for $4 \mathrm{~h}$ with a minimum of 10 concentrations; a range finding experiment plus a minimum of two repeated experiments were conducted. The biological metric was the average \%Tail DNA value; a regression analysis of the SCGE concentration-response curve was conducted to obtain the concentration that induced a 50\%Tail DNA value. The details of SCGE analyses were published. ${ }^{11}$

The concentration-response graphs illustrating the CHO cell SCGE genomic DNA damage of the HANs are presented in Figures S11 to S20. 

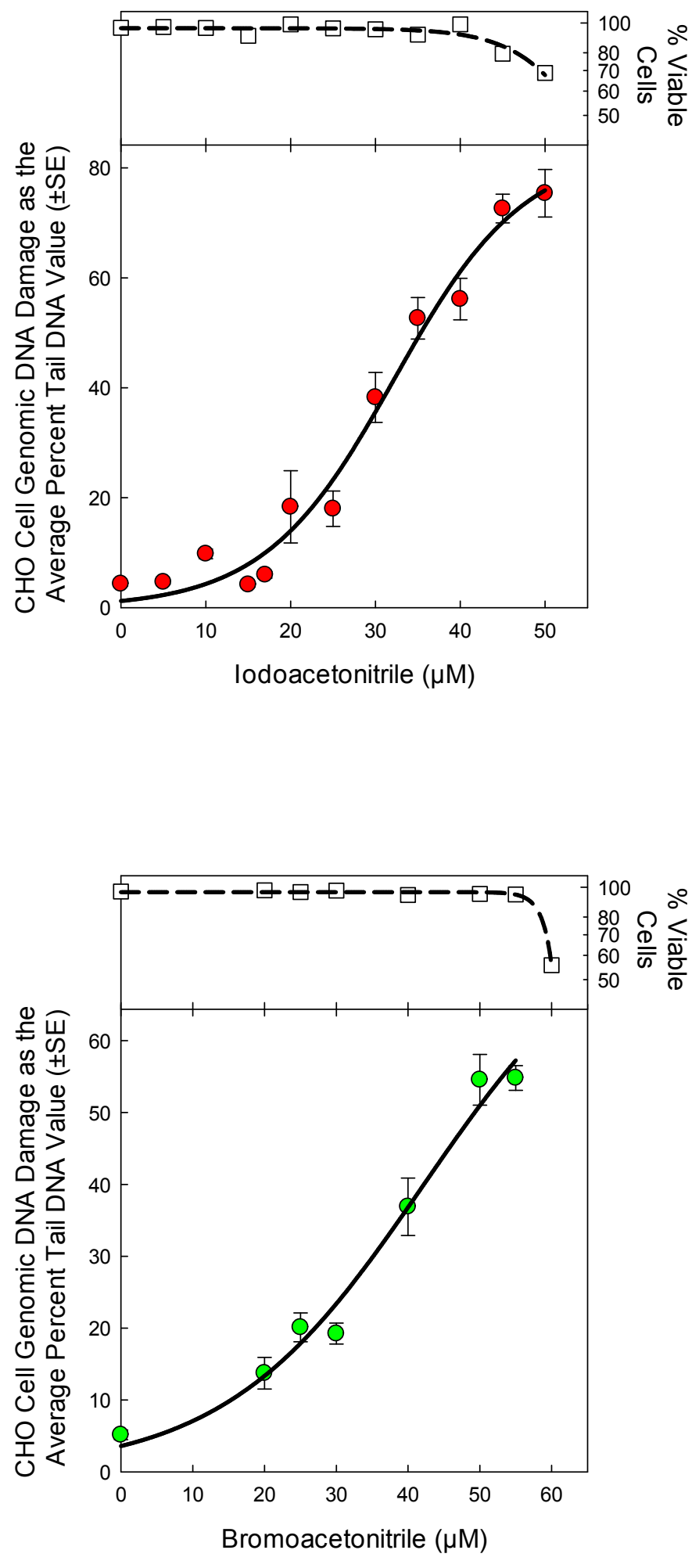

Figure S11. CHO cell genotoxicity concentration-response curve for IAN. The top panel illustrates the acute cytotoxicity and the bottom panel presents the genotoxicity as the Mean $( \pm \mathrm{SE}) 50 \%$ Tail DNA value that was $34.24 \pm 0.76 \mu \mathrm{M}$.
Figure S12. CHO cell genotoxicity concentration-response curve for BAN. The top panel illustrates the acute cytotoxicity and the bottom panel presents the genotoxicity as the Mean $( \pm \mathrm{SE}) 50 \%$ Tail DNA value that was $48.19 \pm 1.06 \mu \mathrm{M}$. 

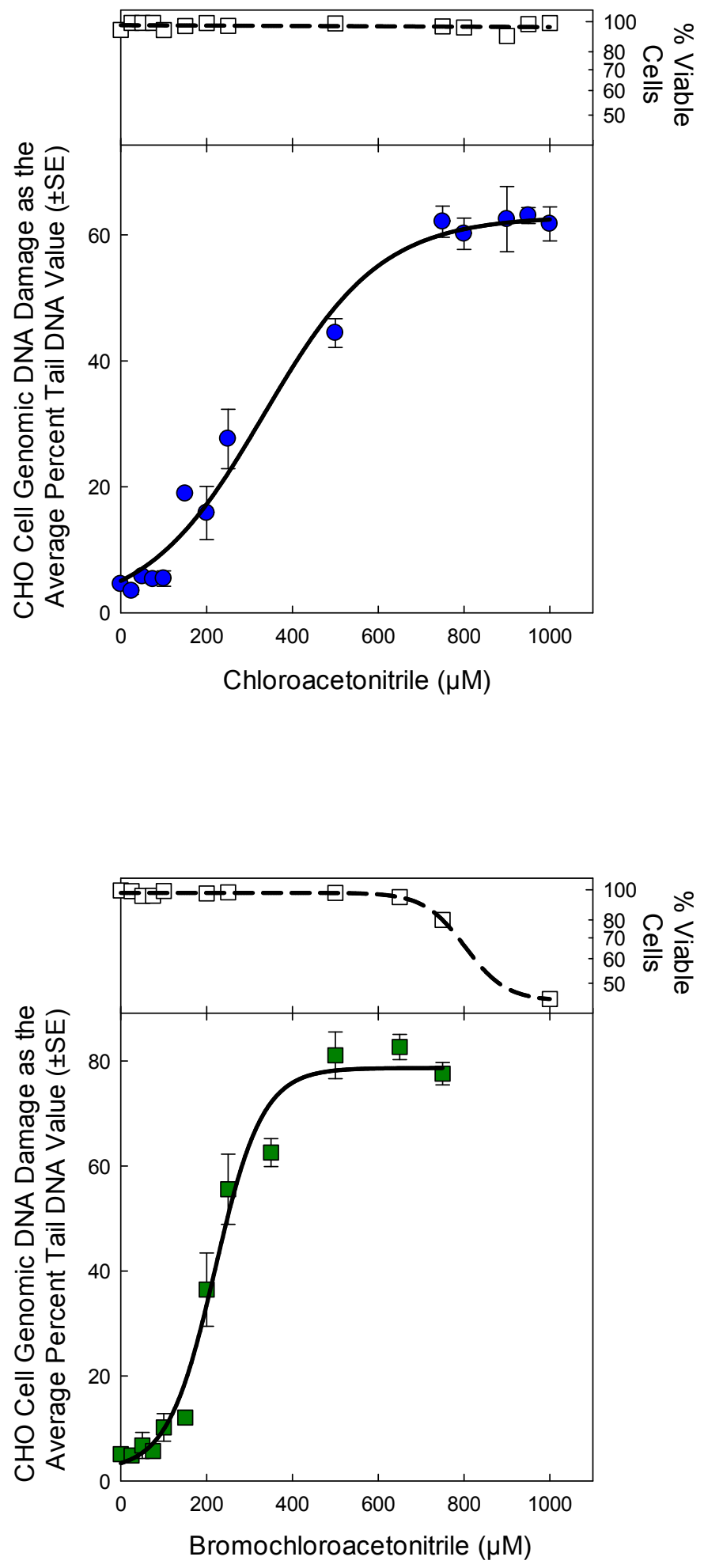

Figure S13. CHO cell genotoxicity concentration-response curve for CAN. The top panel illustrates the acute cytotoxicity and the bottom panel presents the genotoxicity as the Mean $( \pm \mathrm{SE}) 50 \%$ Tail DNA value that was $517.9 \pm 26.2 \mu \mathrm{M}$.
Figure S14. CHO cell genotoxicity concentration-response curve for BCAN. The top panel illustrates the acute cytotoxicity and the bottom panel presents the genotoxicity as the Mean $( \pm \mathrm{SE})$ $50 \%$ Tail DNA value that was $250.1 \pm 12.3 \mu \mathrm{M}$. 

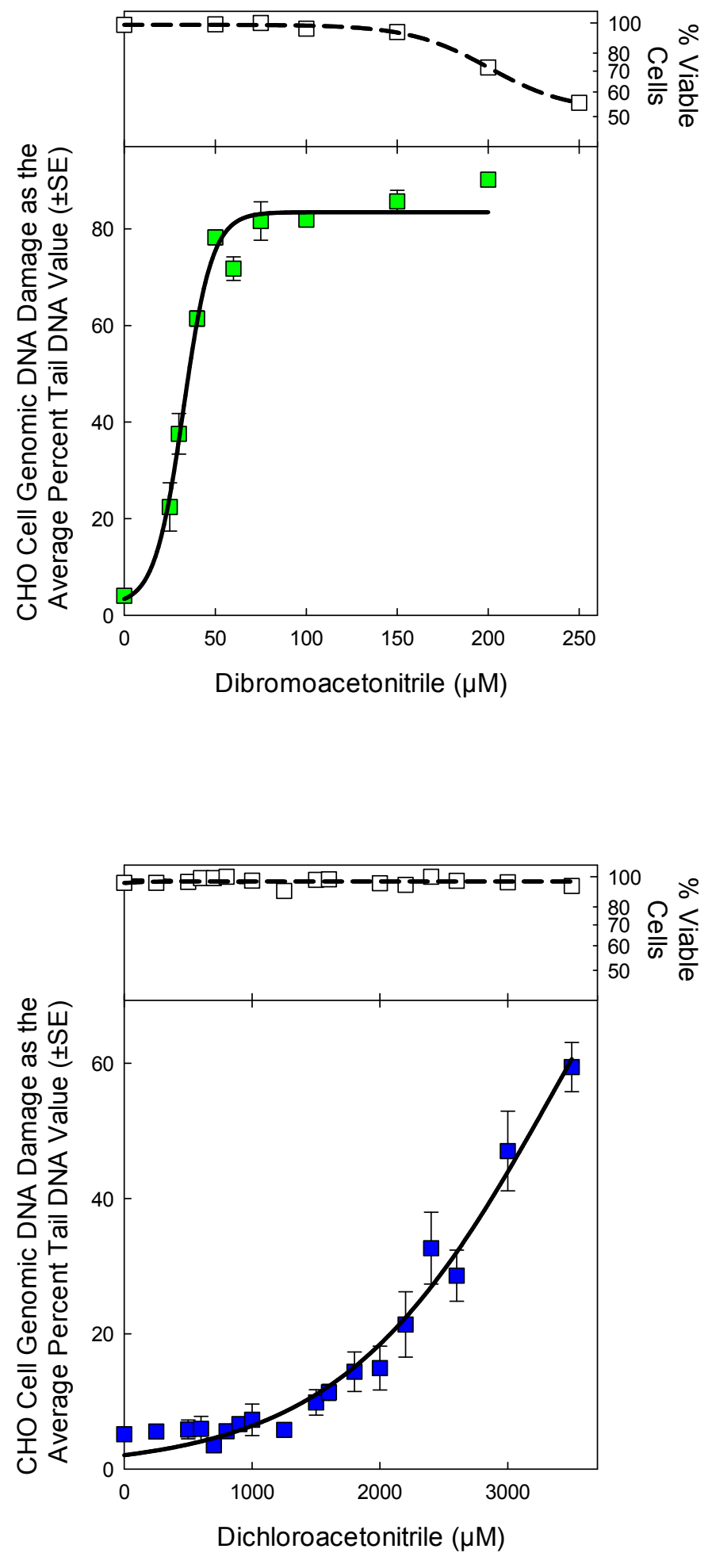

Figure S15. CHO cell genotoxicity concentration-response curve for DBAN. The top panel illustrates the acute cytotoxicity and the bottom panel presents the genotoxicity as the Mean $( \pm \mathrm{SE})$ $50 \%$ Tail DNA value that was $35.50 \pm 0.37 \mu \mathrm{M}$.
Figure S16. CHO cell genotoxicity concentration-response curve for DCAN. The top panel illustrates the acute cytotoxicity and the bottom panel presents the genotoxicity as the Mean $( \pm \mathrm{SE})$ $50 \%$ Tail DNA value that was $3083 \pm 85.4 \mu \mathrm{M}$. 

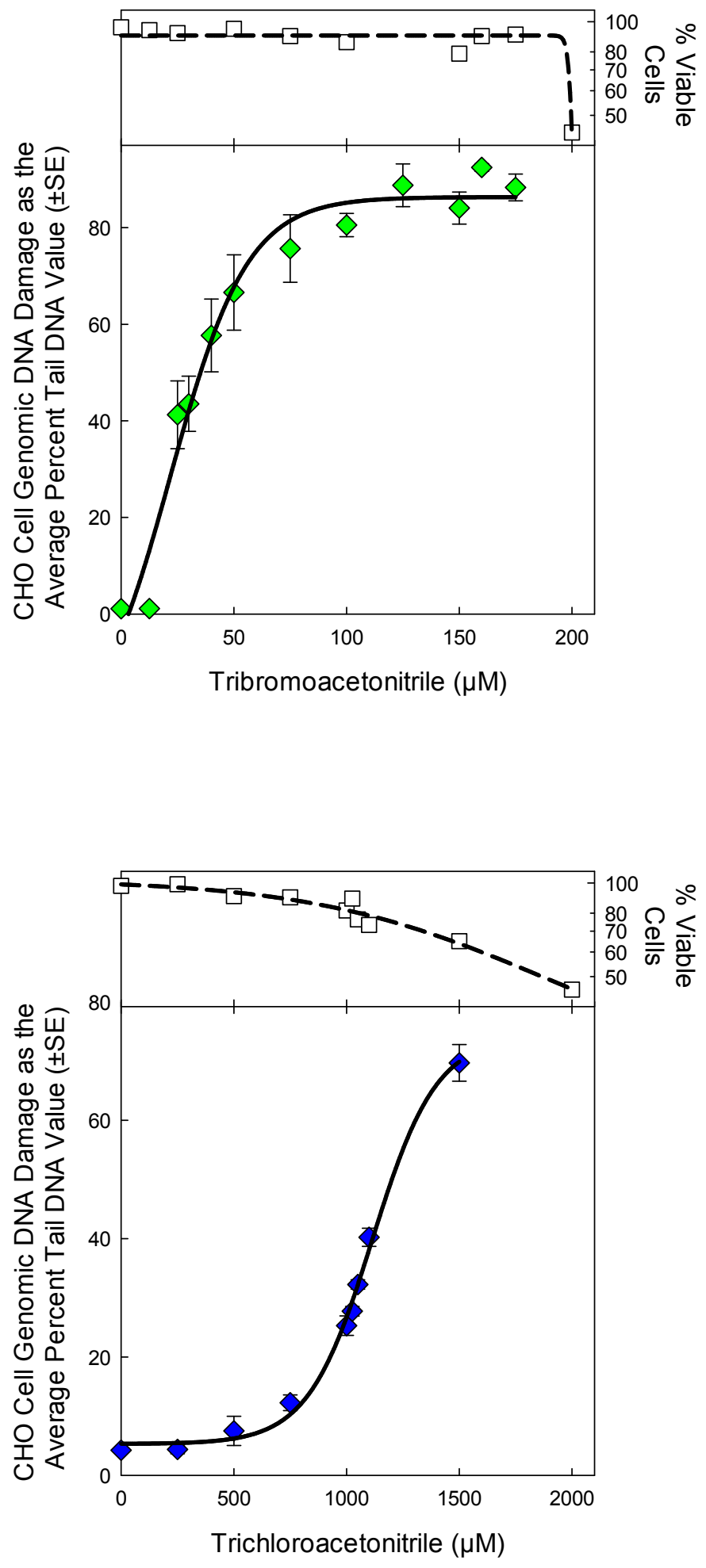

Figure S17. CHO cell genotoxicity concentration-response curve for TBAN. The top panel illustrates the acute cytotoxicity and the bottom panel presents the genotoxicity as the Mean $( \pm \mathrm{SE})$ $50 \%$ Tail DNA value that was $37.76 \pm 3.23 \mu \mathrm{M}$.
Figure S18. CHO cell genotoxicity concentration-response curve for TCAN. The top panel illustrates the acute cytotoxicity and the bottom panel presents the genotoxicity as the Mean $( \pm$ SE) $50 \%$ Tail DNA value that was $1187 \pm 15.2 \mu \mathrm{M}$. 


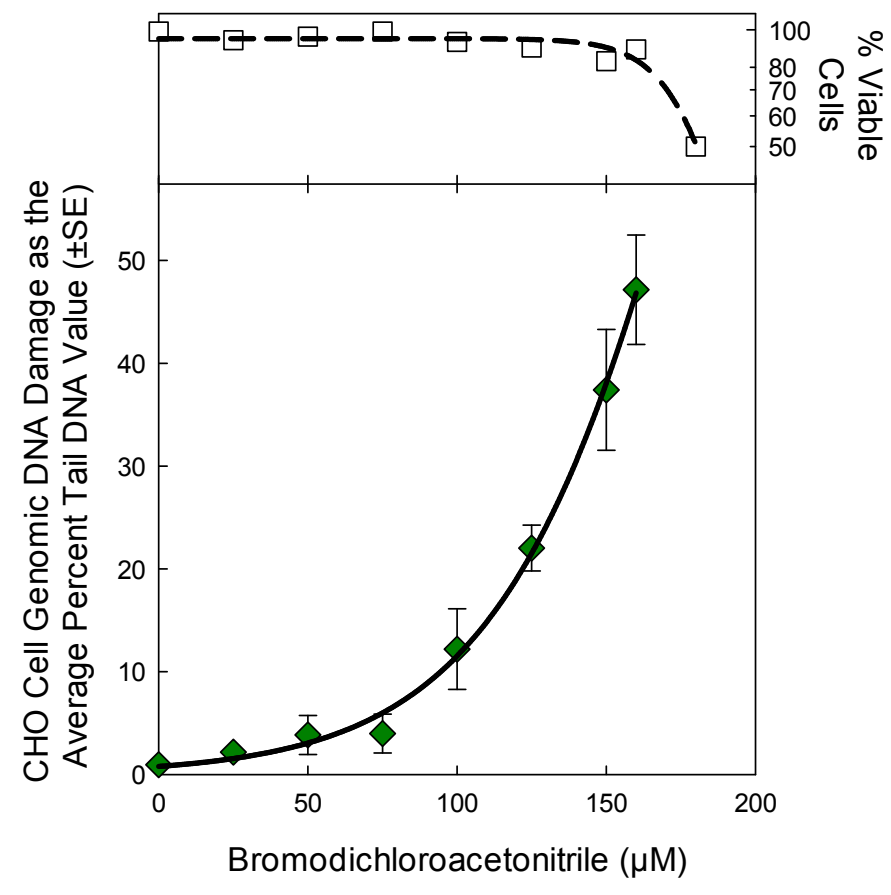

Figure S19. CHO cell genotoxicity concentration-response curve for BDCAN. The top panel illustrates the acute cytotoxicity and the bottom panel presents the genotoxicity as the Mean $( \pm \mathrm{SE})$ $50 \%$ Tail DNA value that was $163.29 \pm 1.45 \mu \mathrm{M}$.

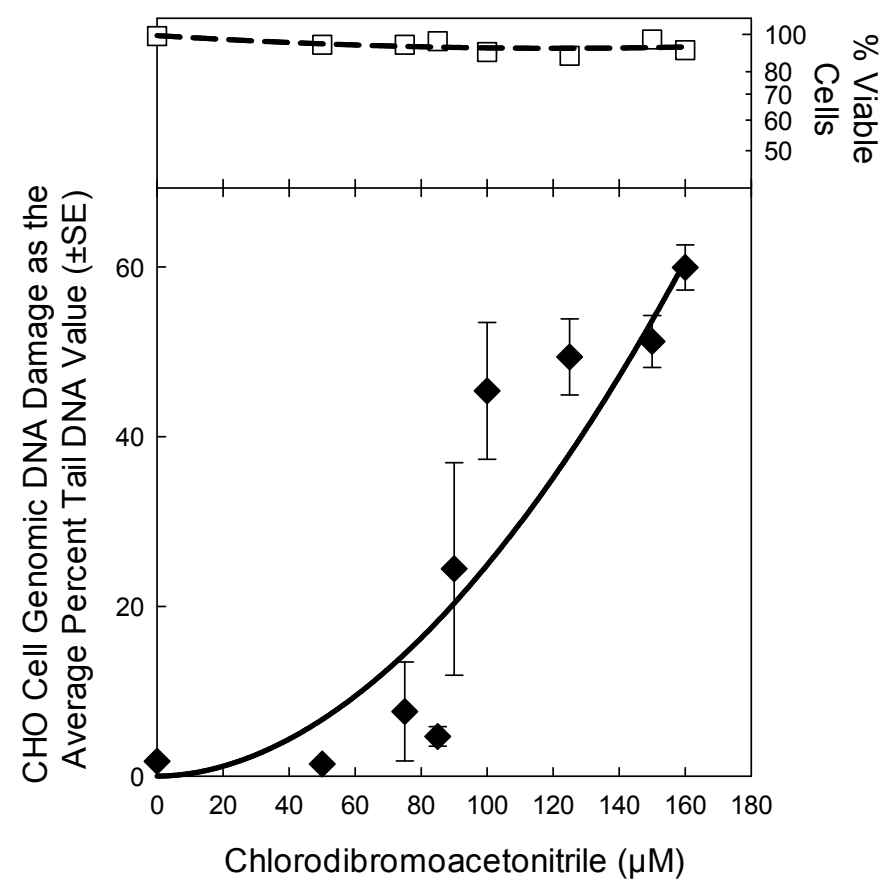

Figure S20. CHO cell genotoxicity concentration-response curve for CDBAN. The top panel illustrates the acute cytotoxicity and the bottom panel presents the genotoxicity as the Mean $( \pm \mathrm{SE})$ $50 \%$ Tail DNA value that was $139.73 \pm 2.89 \mu \mathrm{M}$. 
The ANOVA test statistic to determine if a significant increase in genomic DNA damage (\%TDNA) over their concurrent negative control for the HANs are presented in Tables S6 Table S15.

Table S6. One Way Analysis of Variance: Iodoacetonitrile (IAN) CHO cell genotoxicity SCGE $\%$ Tail DNA.

\begin{tabular}{lrcrrrr} 
Group Name & N & Missing & \multicolumn{1}{c}{ Mean } & Std Dev & SEM & \\
IAN 0 $\mu \mathrm{M}$ & 8 & 0 & 4.325 & 1.105 & 0.391 & \\
IAN 5 & 2 & 0 & 4.656 & 0.507 & 0.358 & \\
IAN 10 & 2 & 0 & 9.779 & 1.234 & 0.872 & \\
IAN 15 & 4 & 0 & 4.194 & 0.862 & 0.431 & \\
IAN 17 & 6 & 0 & 5.968 & 1.444 & 0.590 & \\
IAN 20 & 6 & 0 & 18.331 & 16.081 & 6.565 & \\
IAN 25 & 6 & 0 & 17.993 & 7.881 & 3.217 & \\
IAN 30 & 6 & 0 & 38.251 & 11.131 & 4.544 & \\
IAN 35 & 8 & 0 & 52.653 & 10.681 & 3.776 & \\
IAN 40 & 6 & 0 & 56.148 & 9.226 & 3.766 & \\
IAN 45 & 10 & 0 & 72.612 & 8.229 & 2.602 & \\
IAN 50 & 4 & 0 & 75.403 & 8.648 & 4.324 & \\
& & & & & & \\
Source of Variation & DF & SS & MS & F & P \\
Between Groups & & 11 & 48799.515 & 4436.320 & 57.723 & $<0.001$ \\
Residual & & 56 & 4303.882 & 76.855 & & \\
Total & & 67 & 53103.396 & & &
\end{tabular}

The differences in the mean values among the treatment groups are greater than would be expected by chance; there is a statistically significant difference $(\mathrm{P}=<0.001)$.

Power of performed test with alpha $=0.050: 1.000$

Multiple Comparisons versus Control Group (Holm-Sidak method):

Overall significance level $=0.05$

Comparisons for factor:

$\begin{array}{lcccc}\text { Comparison } & \text { Diff of Means } & \mathbf{t} & \mathbf{P} & \mathbf{P}<\mathbf{0 . 0 5 0} \\ \text { IAN } 0 \mu \mathrm{M} \text { vs. IAN 45 } & 68.287 & 16.421 & <0.001 & \text { Yes } \\ \text { IAN } 0 \mu \mathrm{M} \text { vs. IAN 50 } & 71.079 & 13.240 & <0.001 & \text { Yes } \\ \text { IAN } 0 \mu \mathrm{M} \text { vs. IAN 35 } & 48.328 & 11.025 & <0.001 & \text { Yes } \\ \text { IAN } 0 \mu \mathrm{M} \text { vs. IAN 40 } & 51.824 & 10.946 & <0.001 & \text { Yes } \\ \text { IAN } 0 \mu \mathrm{M} \text { vs. IAN 30 } & 33.926 & 7.166 & <0.001 & \text { Yes } \\ \text { IAN } 0 \mu \mathrm{M} \text { vs. IAN 20 } & 14.006 & 2.958 & 0.027 & \text { Yes } \\ \text { IAN } 0 \mu \mathrm{M} \text { vs. IAN 25 } & 13.668 & 2.887 & 0.027 & \text { Yes } \\ \text { IAN } 0 \mu \mathrm{M} \text { vs. IAN 10 } & 5.454 & 0.787 & 0.898 & \text { No } \\ \text { IAN } 0 \mu \mathrm{M} \text { vs. IAN 17 } & 1.643 & 0.347 & 0.980 & \text { No } \\ \text { IAN } 0 \mu \mathrm{M} \text { vs. IAN 5 } & 0.332 & 0.0479 & 0.999 & \text { No } \\ \text { IAN } 0 \mu \mathrm{M} \text { vs. IAN 15 } & 0.130 & 0.0243 & 0.981 & \text { No }\end{array}$


Table S7. One Way Analysis of Variance: Bromoacetonitrile (BAN) CHO cell genotoxicity SCGE \%Tail DNA.

$\begin{array}{lcccccc}\text { Group Name } & \text { N } & \text { Missing } & \text { Mean } & \text { Std Dev } & \text { SEM } & \\ \text { BAN 0 } \mu \text { M } & 8 & 0 & 5.151 & 1.878 & 0.664 & \\ \text { BAN 20 } & 4 & 0 & 13.727 & 4.414 & 2.207 & \\ \text { BAN 25 } & 6 & 0 & 20.110 & 4.909 & 2.004 & \\ \text { BAN 30 } & 6 & 0 & 19.237 & 3.575 & 1.459 & \\ \text { BAN 40 } & 6 & 0 & 36.887 & 9.771 & 3.989 & \\ \text { BAN 50 } & 7 & 0 & 54.552 & 9.332 & 3.527 & \\ \text { BAN 55 } & 6 & 0 & 54.809 & 4.210 & 1.719 & \\ & & & & & & \\ \text { Source of Variation } & \text { DF } & \text { SS } & \text { MS } & \text { F } & \text { P } \\ \text { Between Groups } & 6 & 15462.675 & 2577.113 & 68.419 & <0.001 \\ \text { Residual } & 36 & 1356.006 & 37.667 & & \\ \text { Total } & 42 & 16818.681 & & & \end{array}$

The differences in the mean values among the treatment groups are greater than would be expected by chance; there is a statistically significant difference $(\mathrm{P}=<0.001)$.

Power of performed test with alpha $=0.050: 1.000$

Multiple Comparisons versus Control Group (Holm-Sidak method):

Overall significance level $=0.05$

Comparisons for factor:

$\begin{array}{lcrcc}\text { Comparison } & \text { Diff of Means } & \mathbf{t} & \mathbf{P} & \mathbf{P}<\mathbf{0 . 0 5 0} \\ \text { BAN 0 } \mu \text { M vs. BAN 50 } & 49.401 & 15.553 & <0.001 & \text { Yes } \\ \text { BAN 0 } \mu \text { M vs. BAN 55 } & 49.658 & 14.982 & <0.001 & \text { Yes } \\ \text { BAN 0 } \mu \text { M vs. BAN 40 } & 31.736 & 9.575 & <0.001 & \text { Yes } \\ \text { BAN 0 } \mu \text { M vs. BAN 25 } & 14.959 & 4.513 & <0.001 & \text { Yes } \\ \text { BAN 0 } \mu \text { M vs. BAN 30 } & 14.086 & 4.250 & <0.001 & \text { Yes } \\ \text { BAN 0 } \mu \text { M vs. BAN 20 } & 8.576 & 2.282 & 0.029 & \text { Yes }\end{array}$


Table S8. One Way Analysis of Variance: Chloroacetonitrile (CAN) CHO cell genotoxicity SCGE \%Tail DNA.

$\begin{array}{lccrrrrl}\text { Group Name } & \text { N } & \text { Missing } & \text { Mean } & \text { Std Dev } & \text { SEM } & & \\ \text { CAN 0 } \mu \text { M } & 5 & 0 & 4.561 & 0.700 & 0.313 & \\ \text { CAN 25 } & 2 & 0 & 3.464 & 0.770 & 0.544 & \\ \text { CAN 50 } & 2 & 0 & 5.774 & 0.000 & 0.000 & \\ \text { CAN 75 } & 2 & 0 & 5.330 & 1.051 & 0.743 & \\ \text { CAN 100 } & 3 & 0 & 5.429 & 2.112 & 1.219 & \\ \text { CAN 150 } & 2 & 0 & 18.919 & 0.000 & 0.000 & \\ \text { CAN 200 } & 4 & 0 & 15.841 & 8.438 & 4.219 & \\ \text { CAN 250 } & 6 & 0 & 27.600 & 11.586 & 4.730 & \\ \text { CAN 500 } & 6 & 0 & 44.428 & 5.585 & 2.280 & \\ \text { CAN 750 } & 6 & 0 & 62.103 & 6.059 & 2.474 & \\ \text { CAN 800 } & 6 & 0 & 60.184 & 6.105 & 2.492 & \\ \text { CAN 900 } & 6 & 0 & 62.503 & 12.683 & 5.178 & \\ \text { CAN 950 } & 2 & 0 & 63.072 & 1.779 & 1.258 & \\ \text { CAN 1000 } & 6 & 0 & 61.750 & 6.611 & 2.699 & \\ \text { Source of Variation } & \text { DF } & \text { SS } & \text { MS } & \text { F } & \text { P } \\ \text { Between Groups } & 13 & 33642.338 & 2587.872 & 46.490 & <0.001 \\ \text { Residual } & & 44 & 2449.285 & 55.666 & & \\ \text { Total } & 57 & 36091.623 & & & \end{array}$

The differences in the mean values among the treatment groups are greater than would be expected by chance; there is a statistically significant difference $(\mathrm{P}=<0.001)$.

Power of performed test with alpha $=0.050: 1.000$

Multiple Comparisons versus Control Group (Holm-Sidak method):

Overall significance level $=0.05$

Comparisons for factor:

$\begin{array}{lcrrc}\text { Comparison } & \text { Diff of Means } & \mathbf{t} & \mathbf{P} & \mathbf{P}<\mathbf{0 . 0 5 0} \\ \text { CAN } 0 \mu \mathrm{M} \text { vs. CAN 900 } & 57.943 & 12.825 & <0.001 & \text { Yes } \\ \text { CAN } 0 \mu \mathrm{M} \text { vs. CAN 750 } & 57.542 & 12.737 & <0.001 & \text { Yes } \\ \text { CAN } 0 \mu \mathrm{M} \text { vs. CAN 1000 } & 57.190 & 12.659 & <0.001 & \text { Yes } \\ \text { CAN 0 } \mu \mathrm{M} \text { vs. CAN 800 } & 55.623 & 12.312 & <0.001 & \text { Yes } \\ \text { CAN 0 } \mu \mathrm{M} \text { vs. CAN 950 } & 58.511 & 9.373 & <0.001 & \text { Yes } \\ \text { CAN 0 } \mu \mathrm{M} \text { vs. CAN 500 } & 39.867 & 8.824 & <0.001 & \text { Yes } \\ \text { CAN } 0 \mu \mathrm{M} \text { vs. CAN 250 } & 23.039 & 5.100 & <0.001 & \text { Yes } \\ \text { CAN } 0 \mu \mathrm{M} \text { vs. CAN 150 } & 14.359 & 2.300 & 0.147 & \text { No } \\ \text { CAN 0 } \mu \mathrm{M} \text { vs. CAN 200 } & 11.280 & 2.254 & 0.138 & \text { No } \\ \text { CAN } 0 \mu \mathrm{M} \text { vs. CAN 50 } & 1.213 & 0.194 & 0.999 & \text { No } \\ \text { CAN 0 } \mu \mathrm{M} \text { vs. CAN 25 } & 1.097 & 0.176 & 0.997 & \text { No } \\ \text { CAN 0 } \mu \mathrm{M} \text { vs. CAN 100 } & 0.868 & 0.159 & 0.984 & \text { No } \\ \text { CAN } 0 \mu \mathrm{M} \text { vs. CAN 75 } & 0.769 & 0.123 & 0.903 & \text { No }\end{array}$


Table S9. One Way Analysis of Variance: Bromochloroacetonitrile (BCAN) CHO cell genotoxicity SCGE \%Tail DNA.

$\begin{array}{lccrrrr}\text { Group Name } & \text { N } & \text { Missing } & \text { Mean } & \text { Std Dev } & \text { SEM } & \\ \text { BCAN 0 } \mu \text { M } & 6 & 0 & 5.093 & 1.420 & 0.580 & \\ \text { BCAN 25 } & 2 & 0 & 4.835 & 0.897 & 0.635 & \\ \text { BCAN 50 } & 2 & 0 & 6.761 & 3.501 & 2.476 & \\ \text { BCAN 75 } & 2 & 0 & 5.700 & 0.731 & 0.517 & \\ \text { BCAN 100 } & 4 & 0 & 10.190 & 5.251 & 2.625 & \\ \text { BCAN 150 } & 6 & 0 & 12.061 & 3.209 & 1.310 & \\ \text { BCAN 200 } & 6 & 0 & 36.449 & 17.118 & 6.988 & \\ \text { BCAN 250 } & 6 & 0 & 55.574 & 16.365 & 6.681 & \\ \text { BCAN 350 } & 6 & 0 & 62.565 & 6.521 & 2.662 & \\ \text { BCAN 500 } & 6 & 0 & 81.086 & 10.892 & 4.447 & \\ \text { BCAN 650 } & 6 & 0 & 82.674 & 5.914 & 2.415 & \\ \text { BCAN 750 } & 4 & 0 & 77.587 & 4.281 & 2.141 & \\ & & & & & & \\ \text { Source of Variation } & \text { DF } & \text { SS } & \text { MS } & \text { F } & \text { P } \\ \text { Between Groups } & 11 & 53426.540 & 4856.958 & 53.455 & <0.001 \\ \text { Residual } & 44 & 3997.866 & 90.861 & & \\ \text { Total } & 55 & 57424.406 & & & \end{array}$

The differences in the mean values among the treatment groups are greater than would be expected by chance; there is a statistically significant difference $(\mathrm{P}=<0.001)$.

Power of performed test with alpha $=0.050: 1.000$

Multiple Comparisons versus Control Group (Holm-Sidak method):

Overall significance level $=0.05$

Comparisons for factor:

$\begin{array}{lcccc}\text { Comparison } & \text { Diff of Means } & \mathbf{t} & \mathbf{P} & \mathbf{P}<\mathbf{0 . 0 5 0} \\ \text { BCAN 0 } \mu \mathrm{M} \text { vs. BCAN 650 } & 77.581 & 14.097 & <0.001 & \text { Yes } \\ \text { BCAN 0 } \mu \mathrm{M} \text { vs. BCAN 500 } & 75.992 & 13.808 & <0.001 & \text { Yes } \\ \text { BCAN 0 } \mu \mathrm{M} \text { vs. BCAN 750 } & 72.493 & 11.782 & <0.001 & \text { Yes } \\ \text { BCAN 0 } \mu \mathrm{M} \text { vs. BCAN 350 } & 57.471 & 10.443 & <0.001 & \text { Yes } \\ \text { BCAN 0 } \mu \mathrm{M} \text { vs. BCAN 250 } & 50.481 & 9.173 & <0.001 & \text { Yes } \\ \text { BCAN 0 } \mu \mathrm{M} \text { vs. BCAN 200 } & 31.356 & 5.698 & <0.001 & \text { Yes } \\ \text { BCAN 0 } \mu \mathrm{M} \text { vs. BCAN 150 } & 6.967 & 1.266 & 0.696 & \text { No } \\ \text { BCAN 0 } \mu \mathrm{M} \text { vs. BCAN 100 } & 5.096 & 0.828 & 0.880 & \text { No } \\ \text { BCAN 0 } \mu \mathrm{M} \text { vs. BCAN 50 } & 1.668 & 0.214 & 0.995 & \text { No } \\ \text { BCAN 0 } \mu \mathrm{M} \text { vs. BCAN 75 } & 0.606 & 0.0779 & 0.996 & \text { No } \\ \text { BCAN 0 } \mu \mathrm{M} \text { vs. BCAN 25 } & 0.258 & 0.0332 & 0.974 & \text { No }\end{array}$


Table S10. One Way Analysis of Variance: Dibromoacetonitrile (DBAN) CHO cell genotoxicity SCGE \%Tail DNA.

$\begin{array}{lrrrrrrl}\text { Group Name } & \text { N } & \text { Missing } & \text { Mean } & \text { Std Dev } & \text { SEM } & & \\ \text { DBAN 0 } \mu \mathrm{M} & 6 & 0 & 4.056 & 1.114 & 0.455 & \\ \text { DBAN 25 } & 2 & 0 & 22.427 & 7.081 & 5.007 & \\ \text { DBAN 30 } & 2 & 0 & 37.559 & 5.935 & 4.197 & \\ \text { DBAN 40 } & 2 & 0 & 61.450 & 2.251 & 1.592 & \\ \text { DBAN 50 } & 8 & 0 & 78.244 & 4.248 & 1.502 & \\ \text { DBAN 60 } & 2 & 0 & 69.927 & 1.935 & 1.368 & \\ \text { DBAN 75 } & 6 & 0 & 81.632 & 9.744 & 3.978 & \\ \text { DBAN 100 } & 12 & 0 & 82.427 & 2.748 & 0.793 & \\ \text { DBAN 150 } & 6 & 0 & 85.688 & 5.653 & 2.308 & \\ \text { DBAN 200 } & 4 & 0 & 90.201 & 1.262 & 0.631 & \\ & & & & & & & \\ \text { Source of Variation } & \text { DF } & \text { SS } & \text { MS } & \text { F } & \text { P } \\ \text { Between Groups } & 9 & 38930.391 & 4325.599 & 182.313 & <0.001 \\ \text { Residual } & 40 & 949.047 & 23.726 & & \\ \text { Total } & 49 & 39879.438 & & & & \end{array}$

The differences in the mean values among the treatment groups are greater than would be expected by chance; there is a statistically significant difference $(\mathrm{P}=<0.001)$.

Power of performed test with alpha $=0.050: 1.000$

Multiple Comparisons versus Control Group (Holm-Sidak method):

Overall significance level $=0.05$

Comparisons for factor:

$\begin{array}{lcccc}\text { Comparison } & \text { Diff of Means } & \mathbf{t} & \mathbf{P} & \mathbf{P}<\mathbf{0 . 0 5 0} \\ \text { DBAN } 0 \mu \mathrm{M} \text { vs. DBAN 100 } & 78.371 & 32.179 & <0.001 & \text { Yes } \\ \text { DBAN } 0 \mu \mathrm{M} \text { vs. DBAN 150 } & 81.632 & 29.027 & <0.001 & \text { Yes } \\ \text { DBAN } 0 \mu \mathrm{M} \text { vs. DBAN 50 } & 74.187 & 28.202 & <0.001 & \text { Yes } \\ \text { DBAN } 0 \mu \mathrm{M} \text { vs. DBAN 75 } & 77.576 & 27.585 & <0.001 & \text { Yes } \\ \text { DBAN } 0 \mu \mathrm{M} \text { vs. DBAN 200 } & 86.145 & 27.398 & <0.001 & \text { Yes } \\ \text { DBAN } 0 \mu \mathrm{M} \text { vs. DBAN 60 } & 65.871 & 16.562 & <0.001 & \text { Yes } \\ \text { DBAN } 0 \mu \mathrm{M} \text { vs. DBAN 40 } & 57.394 & 14.431 & <0.001 & \text { Yes } \\ \text { DBAN } 0 \mu \mathrm{M} \text { vs. DBAN 30 } & 33.503 & 8.424 & <0.001 & \text { Yes } \\ \text { DBAN } 0 \mu \mathrm{M} \text { vs. DBAN 25 } & 18.371 & 4.619 & <0.001 & \text { Yes }\end{array}$


Table S11. One Way Analysis of Variance: Dichloroacetonitrile (DCAN) CHO cell genotoxicity SCGE \%Tail DNA.

$\begin{array}{lccrrrr}\text { Group Name } & \text { N } & \text { Missing } & \text { Mean } & \text { Std Dev } & \text { SEM } & \\ \text { DCAN 0 } \mu \mathrm{M} & 8 & 0 & 5.150 & 1.150 & 0.407 & \\ \text { DCAN 250 } & 2 & 0 & 5.550 & 0.955 & 0.675 & \\ \text { DCAN 500 } & 2 & 0 & 5.859 & 1.964 & 1.389 & \\ \text { DCAN 600 } & 2 & 0 & 5.958 & 2.601 & 1.839 & \\ \text { DCAN 700 } & 2 & 0 & 3.503 & 0.713 & 0.504 & \\ \text { DCAN 800 } & 2 & 0 & 5.582 & 0.906 & 0.640 & \\ \text { DCAN 900 } & 2 & 0 & 6.651 & 0.308 & 0.218 & \\ \text { DCAN 1000 } & 2 & 0 & 7.287 & 3.298 & 2.332 & \\ \text { DCAN 1250 } & 4 & 0 & 5.795 & 1.286 & 0.643 & \\ \text { DCAN 1500 } & 6 & 0 & 9.848 & 4.613 & 1.883 & \\ \text { DCAN 1600 } & 6 & 0 & 11.341 & 2.903 & 1.185 & \\ \text { DCAN 1800 } & 6 & 0 & 14.381 & 7.152 & 2.920 & \\ \text { DCAN 2000 } & 6 & 0 & 14.925 & 7.947 & 3.244 & \\ \text { DCAN 2200 } & 6 & 0 & 21.373 & 11.836 & 4.832 & \\ \text { DCAN 2400 } & 6 & 0 & 32.670 & 13.014 & 5.313 & \\ \text { DCAN 2600 } & 6 & 0 & 28.597 & 9.284 & 3.790 & \\ \text { DCAN 3000 } & 6 & 0 & 47.039 & 14.420 & 5.887 & \\ \text { DCAN 3500 } & 6 & 0 & 59.444 & 8.916 & 3.640 & \\ & & & & & & \\ \text { Source of Variation } & \text { DF } & \text { SS } & \text { MS } & \text { F } & \text { P } \\ \text { Between Groups } & 17 & 21970.765 & 1292.398 & 19.199 & <01 \\ \text { Residual } & & 62 & 4173.536 & 67.315 & & \\ \text { Total } & & 79 & 26144.301 & & & \end{array}$

The differences in the mean values among the treatment groups are greater than would be expected by chance; there is a statistically significant difference $(\mathrm{P}=<0.001)$.

Power of performed test with alpha $=0.050: 1.000$

Multiple Comparisons versus Control Group (Holm-Sidak method):

Overall significance level $=0.05$

Comparisons for factor:

$\begin{array}{lcccc}\text { Comparison } & \text { Diff of Means } & \mathbf{t} & \mathbf{P} & \mathbf{P}<\mathbf{0 . 0 5 0} \\ \text { DCAN } 0 \mu \mathrm{M} \text { vs. DCAN 3500 } & 54.294 & 12.253 & <0.001 & \text { Yes } \\ \text { DCAN } 0 \mu \mathrm{M} \text { vs. DCAN 3000 } & 41.889 & 9.454 & <0.001 & \text { Yes } \\ \text { DCAN } 0 \mu \mathrm{M} \text { vs. DCAN 2400 } & 27.520 & 6.211 & <0.001 & \text { Yes } \\ \text { DCAN } 0 \mu \mathrm{M} \text { vs. DCAN 2600 } & 23.447 & 5.292 & <0.001 & \text { Yes } \\ \text { DCAN } 0 \mu \mathrm{M} \text { vs. DCAN 2200 } & 16.223 & 3.661 & 0.007 & \text { Yes } \\ \text { DCAN } 0 \mu \mathrm{M} \text { vs. DCAN 2000 } & 9.774 & 2.206 & 0.316 & \text { No } \\ \text { DCAN } 0 \mu \mathrm{M} \text { vs. DCAN 1800 } & 9.231 & 2.083 & 0.372 & \text { No } \\ \text { DCAN } 0 \mu \mathrm{M} \text { vs. DCAN 1600 } & 6.191 & 1.397 & 0.840 & \text { No } \\ \text { DCAN } 0 \mu \mathrm{M} \text { vs. DCAN 1500 } & 4.698 & 1.060 & 0.956 & \text { No } \\ \text { DCAN } 0 \mu \mathrm{M} \text { vs. DCAN 1000 } & 2.137 & 0.329 & 1.000 & \text { No } \\ \text { DCAN } 0 \mu \mathrm{M} \text { vs. DCAN 700 } & 1.647 & 0.254 & 1.000 & \text { No } \\ \text { DCAN } 0 \mu \mathrm{M} \text { vs. DCAN 900 } & 1.501 & 0.231 & 1.000 & \text { No } \\ \text { DCAN } 0 \mu \mathrm{M} \text { vs. DCAN 1250 } & 0.645 & 0.128 & 1.000 & \text { No } \\ \text { DCAN } 0 \mu \mathrm{M} \text { vs. DCAN 600 } & 0.808 & 0.125 & 1.000 & \text { No } \\ \text { DCAN } 0 \mu \mathrm{M} \text { vs. DCAN 500 } & 0.709 & 0.109 & 0.999 & \text { No } \\ \text { DCAN } 0 \mu \mathrm{M} \text { vs. DCAN 800 } & 0.432 & 0.0666 & 0.997 & \text { No } \\ \text { DCAN } 0 \mu \mathrm{M} \text { vs. DCAN 250 } & 0.400 & 0.0617 & 0.951 & \text { No }\end{array}$


Table S12. One Way Analysis of Variance: Tribromoacetonitrile (TBAN) CHO cell genotoxicity SCGE \%Tail DNA.

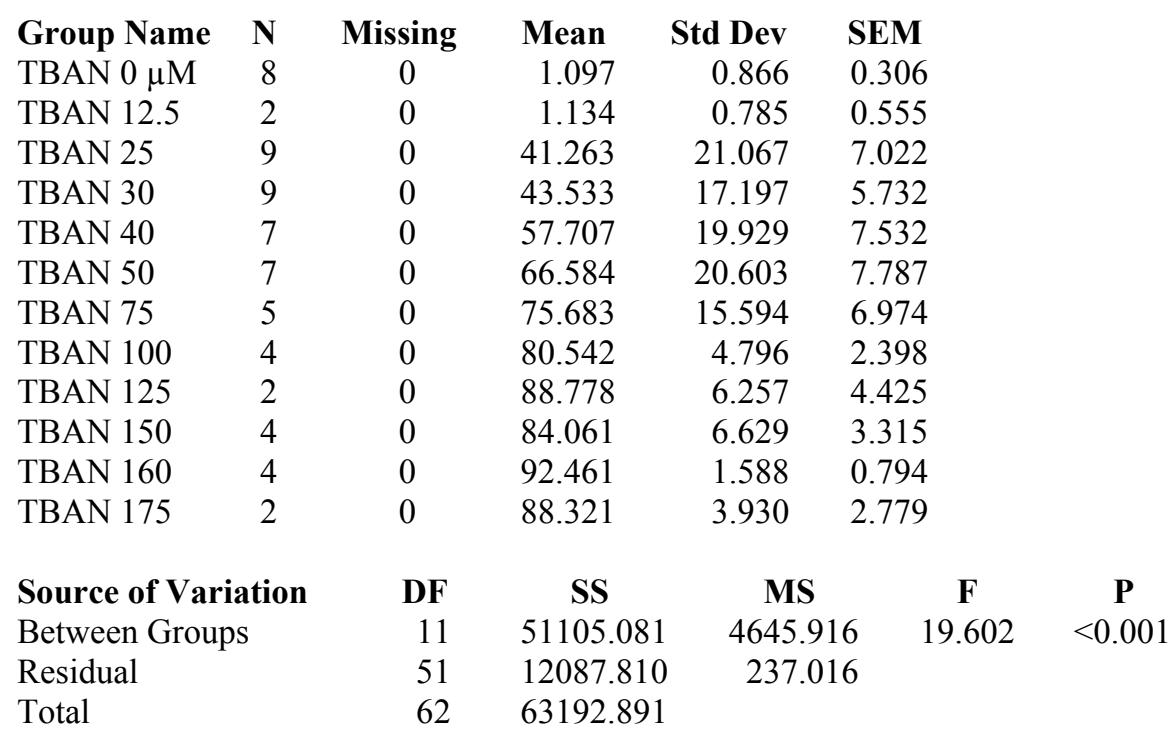

The differences in the mean values among the treatment groups are greater than would be expected by chance; there is a statistically significant difference $(\mathrm{P}=<0.001)$.

Power of performed test with alpha $=0.050: 1.000$

Multiple Comparisons versus Control Group (Holm-Sidak method):

Overall significance level $=0.05$

Comparisons for factor:

$\begin{array}{lcccc}\text { Comparison } & \text { Diff of Means } & \mathbf{t} & \mathbf{P} & \mathbf{P}<\mathbf{0 . 0 5 0} \\ \text { TBAN } 0 \mu \mathrm{M} \text { vs. TBAN 160 } & 91.364 & 9.691 & <0.001 & \text { Yes } \\ \text { TBAN } 0 \mu \mathrm{M} \text { vs. TBAN 150 } & 82.964 & 8.800 & <0.001 & \text { Yes } \\ \text { TBAN } 0 \mu \mathrm{M} \text { vs. TBAN 75 } & 74.586 & 8.498 & <0.001 & \text { Yes } \\ \text { TBAN } 0 \mu \mathrm{M} \text { vs. TBAN 100 } & 79.445 & 8.427 & <0.001 & \text { Yes } \\ \text { TBAN } 0 \mu \mathrm{M} \text { vs. TBAN 50 } & 65.487 & 8.219 & <0.001 & \text { Yes } \\ \text { TBAN } 0 \mu \mathrm{M} \text { vs. TBAN 125 } & 87.681 & 7.204 & <0.001 & \text { Yes } \\ \text { TBAN } 0 \mu \mathrm{M} \text { vs. TBAN 175 } & 87.224 & 7.166 & <0.001 & \text { Yes } \\ \text { TBAN } 0 \mu \mathrm{M} \text { vs. TBAN 40 } & 56.609 & 7.105 & <0.001 & \text { Yes } \\ \text { TBAN } 0 \mu \mathrm{M} \text { vs. TBAN 30 } & 42.436 & 5.673 & <0.001 & \text { Yes } \\ \text { TBAN } 0 \mu \mathrm{M} \text { vs. TBAN 25 } & 40.166 & 5.369 & <0.001 & \text { Yes } \\ \text { TBAN } 0 \mu \mathrm{M} \text { vs. TBAN 12.5 } & 0.0370 & 0.00304 & 0.998 & \text { No }\end{array}$


Table S13. One Way Analysis of Variance: Trichloroacetonitrile (TCAN) CHO cell genotoxicity SCGE \%Tail DNA.

\begin{tabular}{|c|c|c|c|c|c|c|}
\hline Group Name & $\mathbf{N}$ & Missing & Mean & Std Dev & \multicolumn{2}{|l|}{ SEM } \\
\hline TCAN $0 \mu \mathrm{M}$ & 6 & 0 & 4.247 & 0.454 & \multicolumn{2}{|l|}{0.185} \\
\hline TCAN 250 & 2 & 0 & 4.379 & 1.034 & \multicolumn{2}{|l|}{0.731} \\
\hline TCAN 500 & 2 & 0 & 7.509 & 3.486 & \multicolumn{2}{|l|}{2.465} \\
\hline TCAN 750 & 6 & 0 & 12.253 & 3.246 & \multicolumn{2}{|l|}{1.325} \\
\hline TCAN 1000 & 6 & 0 & 25.298 & 4.041 & \multicolumn{2}{|l|}{1.650} \\
\hline TCAN 1025 & 6 & 0 & 27.733 & 1.993 & \multicolumn{2}{|l|}{0.814} \\
\hline TCAN 1050 & 6 & 0 & 32.247 & 1.920 & \multicolumn{2}{|l|}{0.784} \\
\hline TCAN 1100 & 6 & 0 & 40.234 & 3.747 & \multicolumn{2}{|l|}{1.530} \\
\hline TCAN 1500 & 2 & 0 & 69.713 & 4.380 & \multicolumn{2}{|l|}{3.097} \\
\hline \multicolumn{2}{|c|}{ Source of Variation } & DF & SS & MS & \multirow{2}{*}{$\begin{array}{c}\mathbf{F} \\
160.401\end{array}$} & $\mathbf{P}$ \\
\hline \multirow{2}{*}{\multicolumn{2}{|c|}{$\begin{array}{l}\text { Between Groups } \\
\text { Residual }\end{array}$}} & 8 & 10744.004 & 1343.001 & & $<0.001$ \\
\hline & & 33 & 276.302 & & \multirow{2}{*}{373} & \\
\hline Total & & 41 & 11020.306 & & & \\
\hline
\end{tabular}

The differences in the mean values among the treatment groups are greater than would be expected by chance; there is a statistically significant difference $(\mathrm{P}=<0.001)$.

Power of performed test with alpha $=0.050: 1.000$

Multiple Comparisons versus Control Group (Holm-Sidak method):

Overall significance level $=0.05$

Comparisons for factor:

$\begin{array}{lcccc}\text { Comparison } & \text { Diff of Means } & \mathbf{t} & \mathbf{P} & \mathbf{P}<\mathbf{0 . 0 5 0} \\ \text { TCAN } 0 \mu \mathrm{M} \text { vs. TCAN 1500 } & 65.466 & 27.709 & <0.001 & \text { Yes } \\ \text { TCAN } 0 \mu \mathrm{M} \text { vs. TCAN 1100 } & 35.987 & 21.541 & <0.001 & \text { Yes } \\ \text { TCAN } 0 \mu \mathrm{M} \text { vs. TCAN 1050 } & 28.000 & 16.760 & <0.001 & \text { Yes } \\ \text { TCAN } 0 \mu \mathrm{M} \text { vs. TCAN 1025 } & 23.486 & 14.058 & <0.001 & \text { Yes } \\ \text { TCAN } 0 \mu \mathrm{M} \text { vs. TCAN 1000 } & 21.051 & 12.601 & <0.001 & \text { Yes } \\ \text { TCAN 0 } \mu \mathrm{M} \text { vs. TCAN 750 } & 8.006 & 4.792 & <0.001 & \text { Yes } \\ \text { TCAN } 0 \mu \mathrm{M} \text { vs. TCAN 500 } & 3.261 & 1.380 & 0.322 & \text { No } \\ \text { TCAN } 0 \mu \mathrm{M} \text { vs. TCAN 250 } & 0.132 & 0.0557 & 0.956 & \text { No }\end{array}$


Table S14. One Way Analysis of Variance: Bromodichloroacetonitrile (BDCAN) CHO cell genotoxicity SCGE \%Tail DNA.

$\begin{array}{lcccclll}\text { Group Name } & \text { N } & \text { Missing } & \text { Mean } & \text { Std Dev } & \text { SEM } & \\ \text { 0 BDCAN } \mu \text { M } & 6 & 0 & 0.945 & 0.513 & 0.209 & \\ \text { 25 BDCAN } & 2 & 0 & 2.175 & 0.0288 & 0.0204 & \\ \text { 50 BDCAN } & 2 & 0 & 3.850 & 2.681 & 1.895 & \\ \text { 75 BDCAN } & 2 & 0 & 3.979 & 2.673 & 1.890 & \\ \text { 100 BDCAN } & 6 & 0 & 12.192 & 9.598 & 3.919 & \\ \text { 125 BDCAN } & 6 & 0 & 22.021 & 5.456 & 2.227 & \\ \text { 150 BDCAN } & 6 & 0 & 37.414 & 14.384 & 5.872 & \\ \text { 160 BDCAN } & 7 & 0 & 47.150 & 14.066 & 5.316 & \\ & & & & & & \text { F } & \text { P } \\ \text { Source of Variation } & \text { DF } & \text { SS } & \text { MS } & \text { F } \\ \text { Between Groups } & 7 & 11162.753 & 1594.679 & 16.245 & <0.001 \\ \text { Residual } & & 29 & 2846.767 & 98.164 & & \\ \text { Total } & 36 & 14009.520 & & & \end{array}$

The differences in the mean values among the treatment groups are greater than would be expected by chance; there is a statistically significant difference $(\mathrm{P}=<0.001)$.

Power of performed test with alpha $=0.050: 1.000$

Multiple Comparisons versus Control Group (Holm-Sidak method):

Overall significance level $=0.05$

Comparisons for factor:

$\begin{array}{lcccc}\text { Comparison } & \text { Diff of Means } & \mathbf{t} & \mathbf{P} & \mathbf{P}<\mathbf{0 . 0 5 0} \\ \text { 0 BDCAN } \mu \mathrm{M} \text { vs. 160 BDCAN } & 46.205 & 8.382 & <0.001 & \text { Yes } \\ \text { 0 BDCAN } \mu \mathrm{M} \text { vs. 150 BDCAN } & 36.469 & 6.375 & <0.001 & \text { Yes } \\ \text { 0 BDCAN } \mu \mathrm{M} \text { vs. 125 BDCAN } & 21.076 & 3.684 & 0.005 & \text { Yes } \\ \text { 0 BDCAN } \mu \mathrm{M} \text { vs. 100 BDCAN } & 11.247 & 1.966 & 0.216 & \text { No } \\ \text { 0 BDCAN } \mu \mathrm{M} \text { vs. 75 BDCAN } & 3.035 & 0.375 & 0.976 & \text { No } \\ \text { 0 BDCAN } \mu \mathrm{M} \text { vs. 50 BDCAN } & 2.905 & 0.359 & 0.923 & \text { No } \\ \text { 0 BDCAN } \mu \mathrm{M} \text { vs. 25 BDCAN } & 1.230 & 0.152 & 0.880 & \text { No }\end{array}$


Table S15. One Way Analysis of Variance: Chlorodibromoacetonitrile (CDBAN) CHO cell genotoxicity SCGE \%Tail DNA.

\begin{tabular}{|c|c|c|c|c|c|c|}
\hline Group Name & $\mathbf{N}$ & Missing & Mean & Std Dev & \multicolumn{2}{|l|}{ SEM } \\
\hline $0 \mu \mathrm{M}$ CDBAN & 7 & 0 & 1.756 & 0.674 & 0.255 & \\
\hline 50 CDBAN & 3 & 0 & 1.427 & 0.496 & 0.286 & \\
\hline 75 CDBAN & 3 & 0 & 7.617 & 10.102 & \multicolumn{2}{|l|}{5.832} \\
\hline 85 CDBAN & 4 & 0 & 4.677 & 2.303 & \multicolumn{2}{|l|}{1.152} \\
\hline 90 CDBAN & 2 & 0 & 24.425 & 17.715 & \multicolumn{2}{|l|}{12.526} \\
\hline 100 CDBAN & 6 & 0 & 45.406 & 19.735 & \multicolumn{2}{|l|}{8.057} \\
\hline 125 CDBAN & 9 & 0 & 49.414 & 13.436 & \multicolumn{2}{|l|}{4.479} \\
\hline 150 CDBAN & 10 & 0 & 51.228 & 9.701 & \multicolumn{2}{|l|}{3.068} \\
\hline 160 CDBAN & 6 & 0 & 59.954 & 6.529 & \multicolumn{2}{|l|}{2.665} \\
\hline \multicolumn{2}{|c|}{ Source of Variation } & DF & SS & MS & \multirow{3}{*}{$\begin{array}{c}\mathbf{F} \\
26.812\end{array}$} & $\mathbf{P}$ \\
\hline \multicolumn{2}{|c|}{ Between Groups } & 8 & 26100.281 & 3262.535 & & $<0.001$ \\
\hline \multicolumn{2}{|c|}{ Residual } & 41 & 4988.874 & \multirow{2}{*}{\multicolumn{2}{|c|}{121.680}} & \\
\hline Total & & 49 & 31089.156 & & & \\
\hline
\end{tabular}

The differences in the mean values among the treatment groups are greater than would be expected by chance; there is a statistically significant difference $(\mathrm{P}=<0.001)$.

Power of performed test with alpha $=0.050: 1.000$

Multiple Comparisons versus Control Group (Holm-Sidak method):

Overall significance level $=0.05$

Comparisons for factor:

$\begin{array}{lcccc}\text { Comparison } & \text { Diff of Means } & \mathbf{t} & \mathbf{P} & \mathbf{P}<\mathbf{0 . 0 5 0} \\ 0 \mu \mathrm{M} \text { CDBAN vs. 160 CDBAN } & 58.198 & 9.483 & <0.001 & \text { Yes } \\ 0 \mu \text { M CDBAN vs. 150 CDBAN } & 49.472 & 9.101 & <0.001 & \text { Yes } \\ 0 \mu \text { M CDBAN vs. 125 CDBAN } & 47.658 & 8.573 & <0.001 & \text { Yes } \\ 0 \mu \text { M CDBAN vs. 100 CDBAN } & 43.650 & 7.113 & <0.001 & \text { Yes } \\ 0 \mu \text { CDBAN vs. 90 CDBAN } & 22.669 & 2.563 & 0.055 & \text { No } \\ 0 \mu \text { M CDBAN vs. 75 CDBAN } & 5.861 & 0.770 & 0.830 & \text { No } \\ 0 \mu \text { M CDBAN vs. 85 CDBAN } & 2.921 & 0.422 & 0.894 & \text { No } \\ 0 \mu \text { M CDBAN vs. 50 CDBAN } & 0.329 & 0.0432 & 0.966 & \text { No }\end{array}$


Table S16. One Way Analysis of Variance: 10 HAN GTI Comparisons.

\begin{tabular}{|c|c|c|c|c|c|c|}
\hline Group Name & $\mathbf{N}$ & Missing & Mean & Std Dev & SEM & \\
\hline IAN GTI & 11 & 0 & 29.347 & 2.099 & 0.633 & \\
\hline BAN GTI & 11 & 0 & 20.854 & 1.572 & 0.474 & \\
\hline DBAN GTI & 15 & 0 & 28.210 & 1.141 & 0.295 & \\
\hline BCAN GTI & 11 & 0 & 4.110 & 0.752 & 0.227 & \\
\hline TBAN GTI & 11 & 0 & 28.372 & 7.550 & 2.276 & \\
\hline CAN GTI & 11 & 0 & 1.997 & 0.444 & 0.134 & \\
\hline DCAN GTI & 11 & 0 & 0.327 & 0.0292 & 0.00881 & \\
\hline TCAN GTI & 11 & 0 & 0.844 & 0.0342 & 0.0103 & \\
\hline BDCAN GTI & 11 & 0 & 6.129 & 0.180 & 0.0544 & \\
\hline CDBAN GTI & 11 & 0 & 7.187 & 0.484 & 0.146 & \\
\hline \multicolumn{2}{|c|}{ Source of Variation } & DF & SS & MS & $\mathbf{F}$ & $\mathbf{P}$ \\
\hline \multicolumn{2}{|c|}{ Between Groups } & 9 & 16162.572 & 1795.841 & 279.887 & $<0.001$ \\
\hline \multicolumn{2}{|c|}{ Residual } & 104 & 667.295 & 6.416 & & \\
\hline Total & & 113 & 16829.867 & & & \\
\hline
\end{tabular}

The differences in the mean values among the treatment groups are greater than would be expected by chance; there is a statistically significant difference $(\mathrm{P}=<0.001)$.. Power of performed test with alpha $=0.050: 1.000$

All Pairwise Multiple Comparison Procedures (Holm-Sidak method):

Overall significance level $=0.05$

Comparisons for factor:

$\begin{array}{lcccc}\text { Comparison } & \text { Diff of Means } & \mathbf{t} & \mathbf{P} & \mathbf{P}<\mathbf{0 . 0 5 0} \\ \text { DBAN GTI vs. DCAN GTI } & 27.883 & 27.730 & <0.001 & \text { Yes } \\ \text { DBAN GTI vs. TCAN GTI } & 27.366 & 27.216 & <0.001 & \text { Yes } \\ \text { IAN GTI vs. DCAN GTI } & 29.020 & 26.868 & <0.001 & \text { Yes } \\ \text { IAN GTI vs. TCAN GTI } & 28.503 & 26.390 & <0.001 & \text { Yes } \\ \text { DBAN GTI vs. CAN GTI } & 26.213 & 26.069 & <0.001 & \text { Yes } \\ \text { TBAN GTI vs. DCAN GTI } & 28.045 & 25.965 & <0.001 & \text { Yes } \\ \text { TBAN GTI vs. TCAN GTI } & 27.528 & 25.487 & <0.001 & \text { Yes } \\ \text { IAN GTI vs. CAN GTI } & 27.350 & 25.322 & <0.001 & \text { Yes } \\ \text { TBAN GTI vs. CAN GTI } & 26.375 & 24.419 & <0.001 & \text { Yes } \\ \text { DBAN GTI vs. BCAN GTI } & 24.100 & 23.967 & <0.001 & \text { Yes } \\ \text { IAN GTI vs. BCAN GTI } & 25.237 & 23.365 & <0.001 & \text { Yes } \\ \text { TBAN GTI vs. BCAN GTI } & 24.262 & 22.463 & <0.001 & \text { Yes } \\ \text { DBAN GTI vs. BDCAN GTI } & 22.081 & 21.960 & <0.001 & \text { Yes } \\ \text { IAN GTI vs. BDCAN GTI } & 23.218 & 21.496 & <0.001 & \text { Yes } \\ \text { DBAN GTI vs. CDBAN GTI } & 21.023 & 20.908 & <0.001 & \text { Yes } \\ \text { TBAN GTI vs. BDCAN GTI } & 22.243 & 20.593 & <0.001 & \text { Yes } \\ \text { IAN GTI vs. CDBAN GTI } & 22.160 & 20.517 & <0.001 & \text { Yes } \\ \text { TBAN GTI vs. CDBAN GTI } & 21.185 & 19.614 & <0.001 & \text { Yes } \\ \text { BAN GTI vs. DCAN GTI } & 20.527 & 19.005 & <0.001 & \text { Yes } \\ \text { BAN GTI vs. TCAN GTI } & 20.010 & 18.526 & <0.001 & \text { Yes } \\ \text { BAN GTI vs. CAN GTI } & 18.857 & 17.458 & <0.001 & \text { Yes } \\ \text { BAN GTI vs. BCAN GTI } & 16.744 & 15.502 & <0.001 & \text { Yes } \\ \text { BAN GTI vs. BDCAN GTI } & 14.725 & 13.633 & <0.001 & \text { Yes } \\ \text { BAN GTI vs. CDBAN GTI } & 13.667 & 12.654 & <0.001 & \text { Yes } \\ \text { IAN GTI vs. BAN GTI } & 8.493 & 7.863 & <0.001 & \text { Yes } \\ \text { DBAN GTI vs. BAN GTI } & 7.356 & 7.316 & <0.001 & \text { Yes } \\ \text { TBAN GTI vs. BAN GTI } & 7.518 & 6.961 & <0.001 & \text { Yes } \\ \text { CDBAN GTI vs. DCAN GTI } & 6.860 & 6.351 & <0.001 & \text { Yes }\end{array}$




$\begin{array}{llrrr}\text { CDBAN GTI vs. TCAN GTI } & 6.343 & 5.873 & <0.001 & \text { Yes } \\ \text { BDCAN GTI vs. DCAN GTI } & 5.802 & 5.372 & <0.001 & \text { Yes } \\ \text { BDCAN GTI vs. TCAN GTI } & 5.285 & 4.893 & <0.001 & \text { Yes } \\ \text { CDBAN GTI vs. CAN GTI } & 5.190 & 4.805 & <0.001 & \text { Yes } \\ \text { BDCAN GTI vs. CAN GTI } & 4.132 & 3.826 & 0.003 & \text { Yes } \\ \text { BCAN GTI vs. DCAN GTI } & 3.783 & 3.503 & 0.008 & \text { Yes } \\ \text { BCAN GTI vs. TCAN GTI } & 3.266 & 3.024 & 0.034 & \text { Yes } \\ \text { CDBAN GTI vs. BCAN GTI } & 3.076 & 2.848 & 0.050 & \text { Yes } \\ \text { BCAN GTI vs. CAN GTI } & 2.113 & 1.957 & 0.388 & \text { No } \\ \text { BDCAN GTI vs. BCAN GTI } & 2.019 & 1.869 & 0.413 & \text { No } \\ \text { CAN GTI vs. DCAN GTI } & 1.670 & 1.546 & 0.608 & \text { No } \\ \text { IAN GTI vs. DBAN GTI } & 1.137 & 1.131 & 0.837 & \text { No } \\ \text { CAN GTI vs. TCAN GTI } & 1.153 & 1.068 & 0.817 & \text { No } \\ \text { CDBAN GTI vs. BDCAN GTI } & 1.058 & 0.979 & 0.798 & \text { No } \\ \text { IAN GTI vs. TBAN GTI } & 0.975 & 0.903 & 0.748 & \text { No } \\ \text { TCAN GTI vs. DCAN GTI } & 0.517 & 0.479 & 0.865 & \text { No } \\ \text { TBAN GTI vs. DBAN GTI } & 0.162 & 0.161 & 0.872 & \text { No }\end{array}$

$N$-Acetylcysteine thiol reactivity analyses. The $N$-acetylcysteine (NAC) thiol reactivity high throughput assay is a screen to identify potential adverse biological effects. ${ }^{17-19}$ The cysteine thiol is a reductant against reactive toxicants. ${ }^{20,21}$ HANs were reacted with NAC for 20 min in a volume of $50 \mu \mathrm{L}$, followed by the addition of $50 \mu \mathrm{L}$ of 5,5-dithiobis (2-nitrobenzoic acid) (DTNB, $1 \mathrm{mM}$ ). Each experiment included concurrent negative controls (Tris buffer and NAC), positive controls (Tris buffer, NAC and maleimide), and corresponding blanks to correct for the background $\mathrm{A}_{412}$. After incubation with NAC (20 min, $30^{\circ} \mathrm{C}$ shaking, dark conditions), DTNB was added to quantify the available thiol groups. The microplate was analyzed at $412 \mathrm{~nm}$ using a Molecular Devices Spectramax Paradigm multimode microplate reader after linear shaking of 10 s. The data were saved in an Excel spreadsheet. The $\mathrm{A}_{412}$ values for each well were blankcorrected. The blank-corrected negative controls were averaged. This value was divided into the individual $\mathrm{A}_{412}$ values for each treatment group $\times 100$; the data were expressed as the percent of the concurrent negative controls. Using these normalized data, we generated concentrationresponse curves. Regression analyses were used to calculate the $\mathrm{EC}_{50}$ values, the effective $\mathrm{HAN}$ concentration that induced a reduction in the NAC thiol response by $50 \%$ compared to the concurrent negative controls. 
The concentration-response graphs illustrating the NAC thiol reactivity of the HANs are presented in Figures S21 to S30.

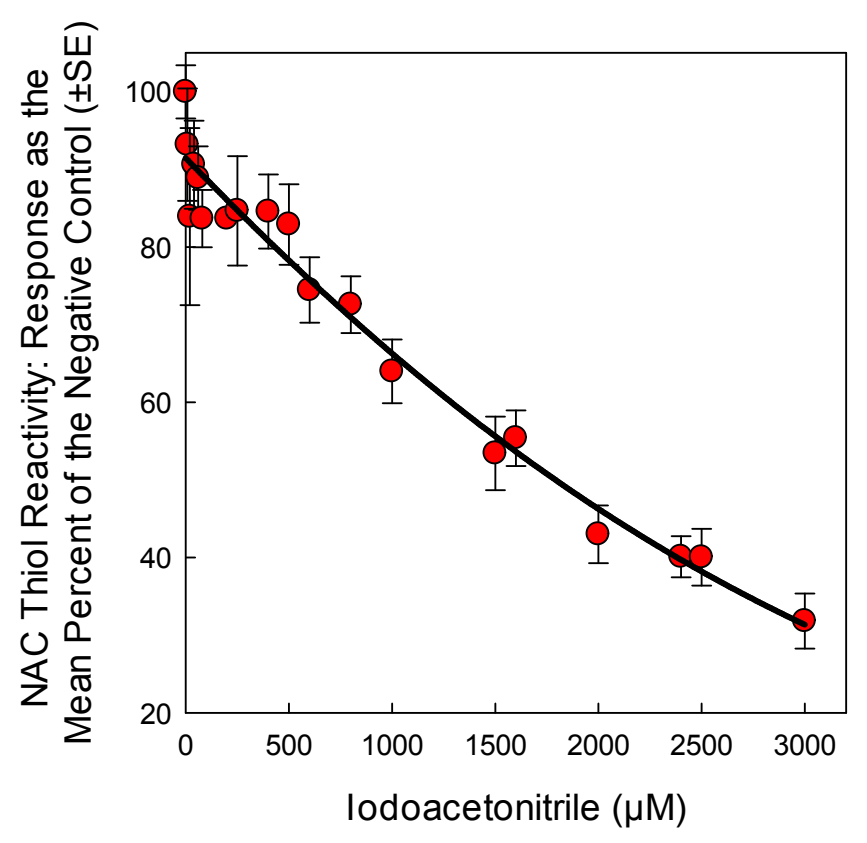

Figure S21. NAC thiol reactivity concentration-response curve for IAN. Mean $( \pm \mathrm{SE}) \mathrm{EC}_{50}$ value was $1714.47 \pm 70.15 \mu \mathrm{M}$.

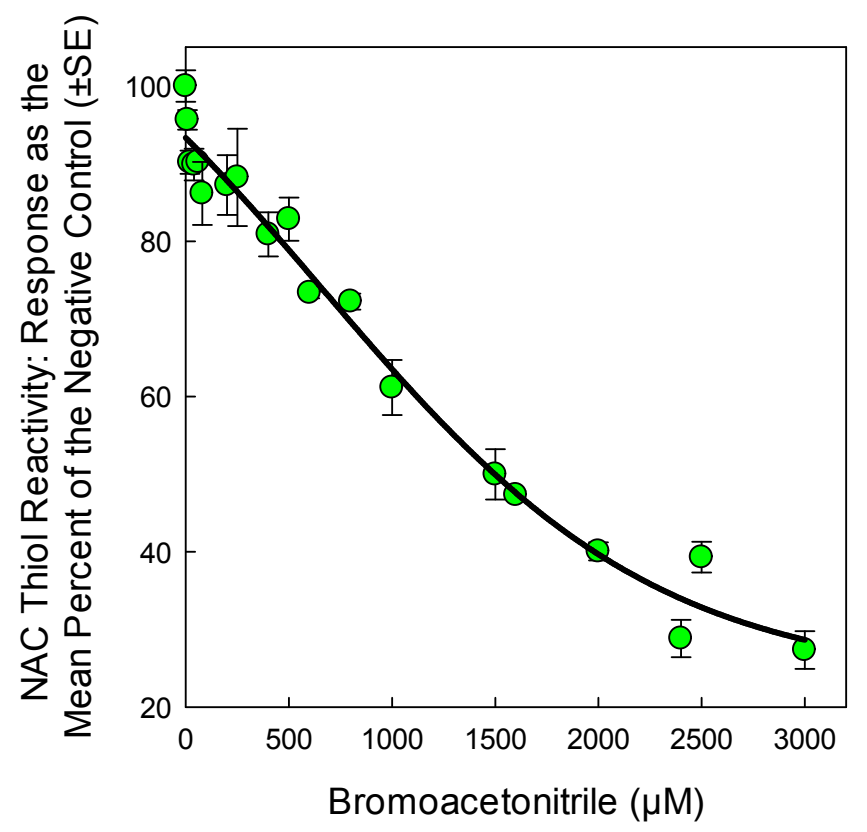

Figure S22. NAC thiol reactivity concentration-response curve for BAN. Mean $( \pm \mathrm{SE}) \mathrm{EC}_{50}$ value was $1502.96 \pm 16.88 \mu \mathrm{M}$. 


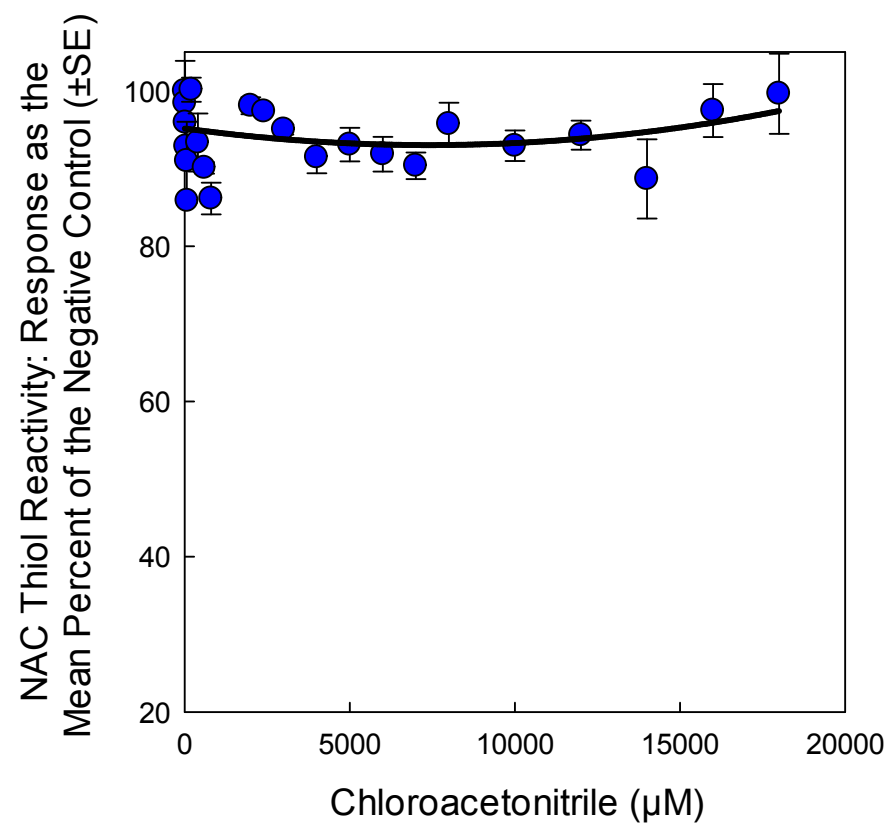

Figure S23. NAC thiol reactivity concentration-response curve for CAN.

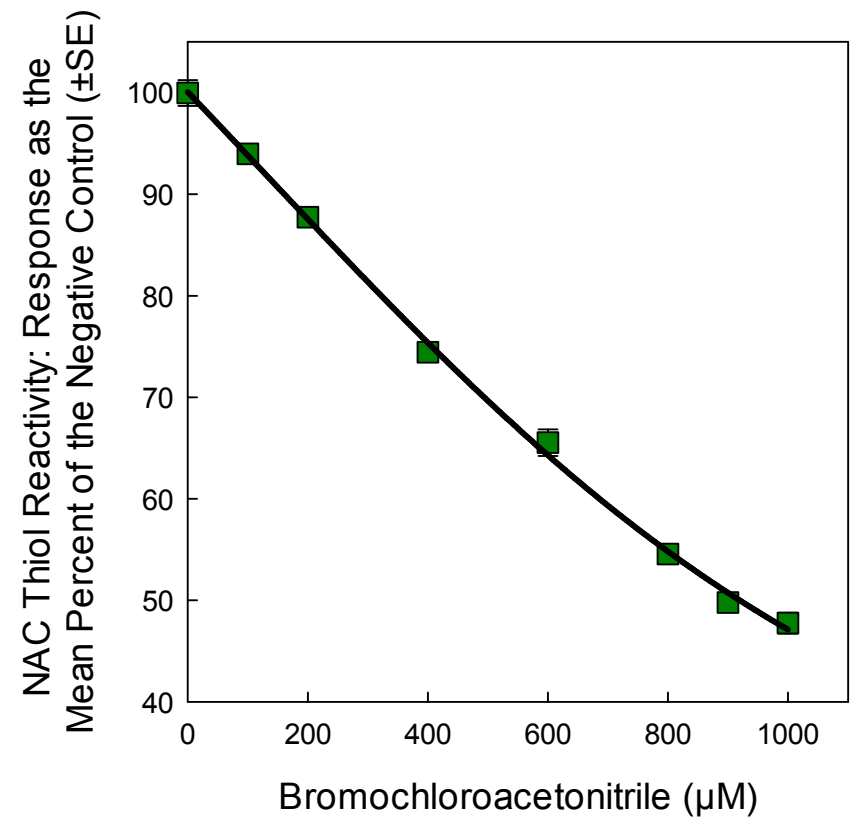

Figure S24. NAC thiol reactivity concentration-response curve for BCAN. Mean $\left( \pm\right.$ SE) $\mathrm{EC}_{50}$ value was $912.84 \pm 11.11 \mu \mathrm{M}$. 

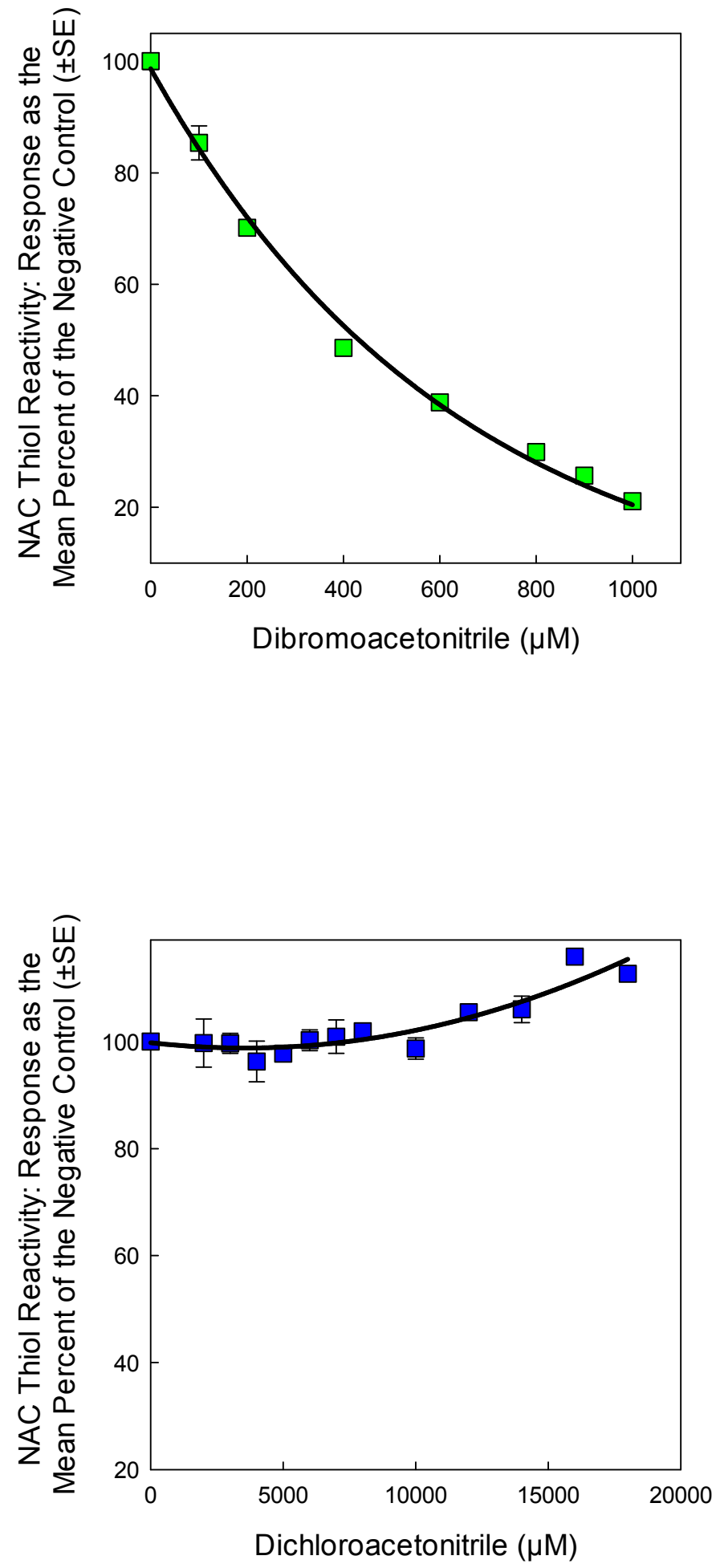

Figure S25. NAC thiol reactivity concentration-response curve for DBAN. Mean $\left( \pm\right.$ SE) EC $_{50}$ value was $403.78 \pm 3.92 \mu \mathrm{M}$.
Figure S26. NAC thiol reactivity concentration-response curve for DCAN. 

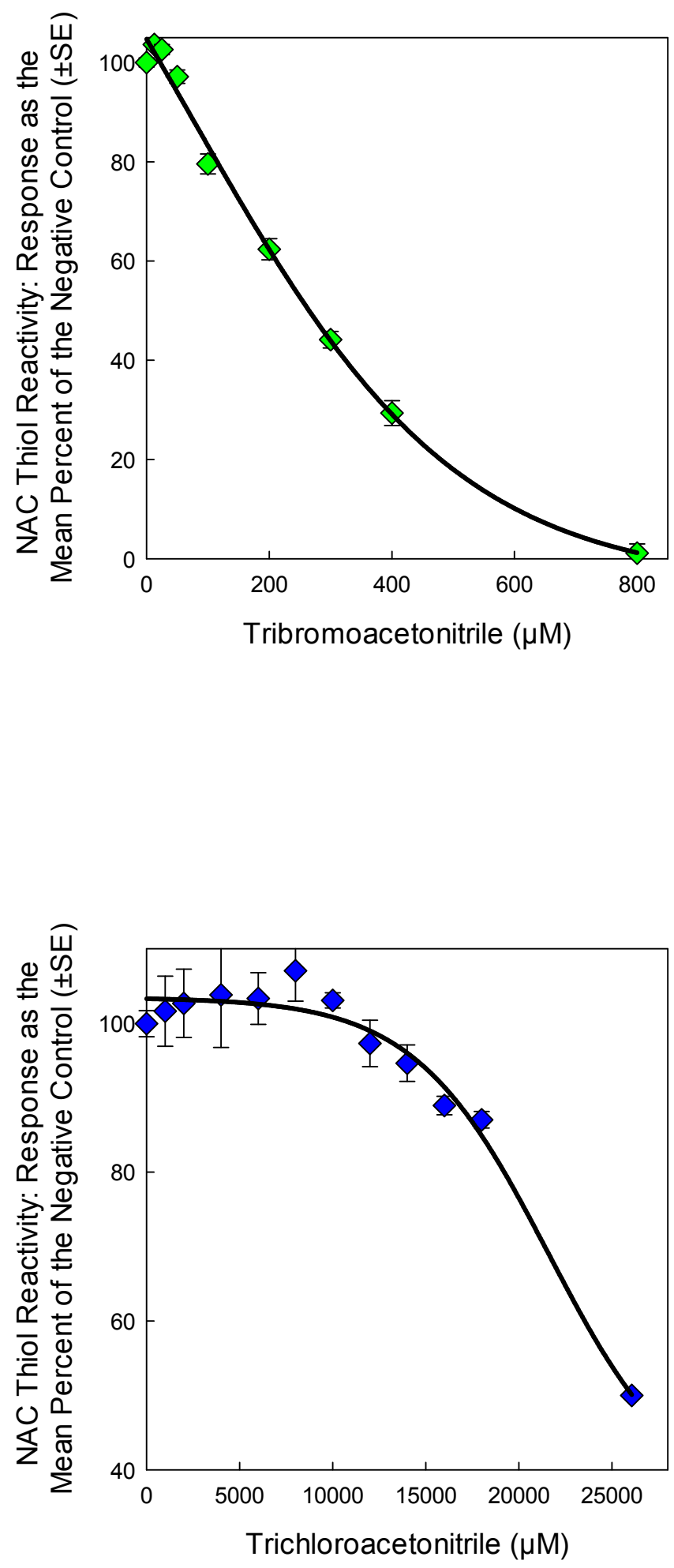

Figure S27. NAC thiol reactivity concentration-response curve for TBAN. Mean $\left( \pm\right.$ SE) EC $_{50}$ value was $263.06 \pm 6.73 \mu \mathrm{M}$.
Figure S28. NAC thiol reactivity concentration-response curve for TCAN. The extrapolated mean $( \pm \mathrm{SE}) \mathrm{EC}_{50}$ value was $26148.67 \pm$ $9.83 \mu \mathrm{M}$. 

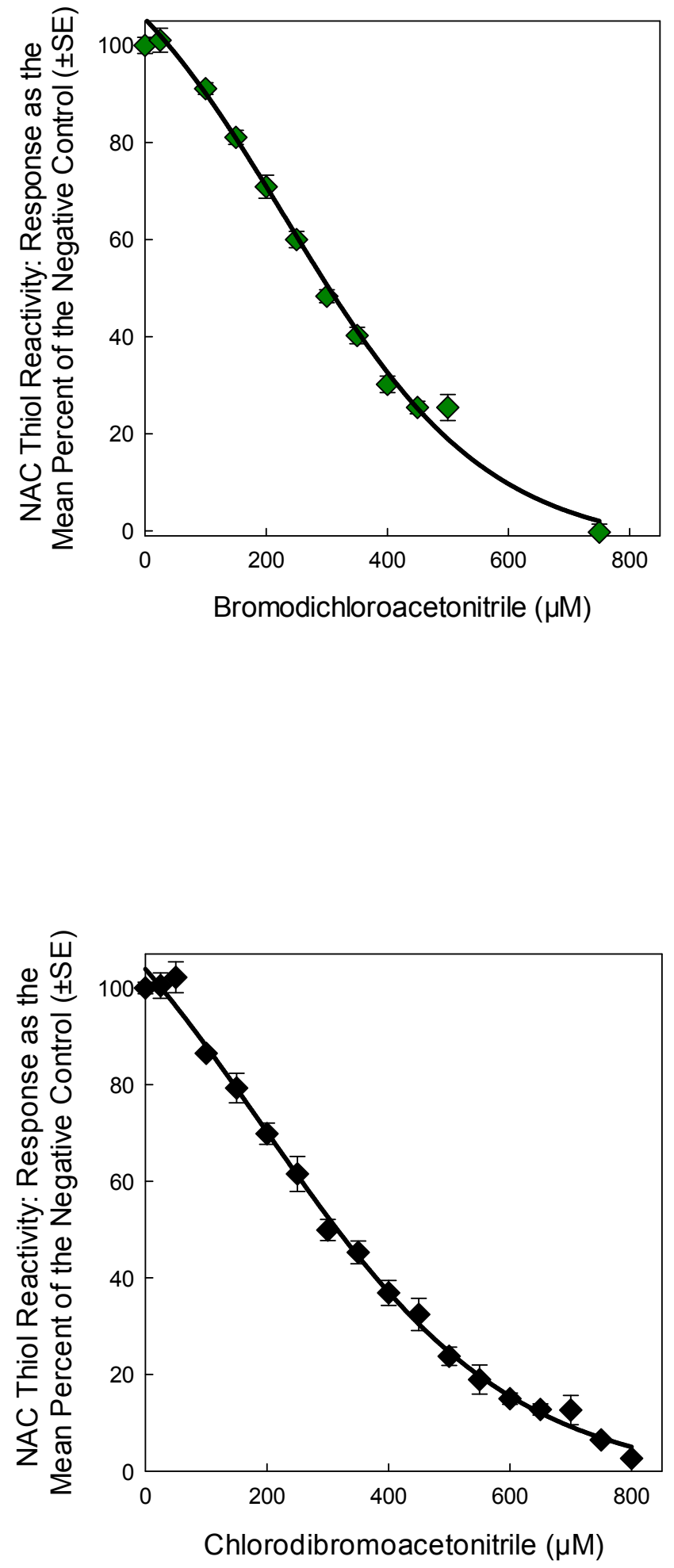

Figure S29. NAC thiol reactivity concentration-response curve for BDCAN. The extrapolated mean $( \pm \mathrm{SE}) \mathrm{EC}_{50}$ value was $302.31 \pm$ $2.04 \mu \mathrm{M}$.
Figure S30. NAC thiol reactivity concentration-response curve for CDBAN. The extrapolated mean $( \pm \mathrm{SE}) \mathrm{EC}_{50}$ value was $314.43 \pm$ $5.74 \mu \mathrm{M}$. 
Table S17. One Way Analysis of Variance: Iodoacetonitrile (IAN) thiol reactivity response as the percent of the negative control.

\begin{tabular}{|c|c|c|c|c|c|c|}
\hline Group Name & $\mathbf{N}$ & Missing & Mean & Std Dev & SEM & \\
\hline 0 IAN & 5 & 0 & 100.000 & 0.000 & 0.000 & \\
\hline $8 \mathrm{IAN}$ & 5 & 0 & 94.640 & 7.924 & 3.544 & \\
\hline 20 IAN & 5 & 0 & 81.632 & 12.495 & 5.588 & \\
\hline 40 IAN & 5 & 0 & 89.466 & 6.206 & 2.775 & \\
\hline 60 IAN & 5 & 0 & 88.077 & 4.471 & 1.999 & \\
\hline 80 IAN & 5 & 0 & 82.926 & 4.048 & 1.810 & \\
\hline 200 IAN & 5 & 0 & 83.655 & 0.000 & 0.000 & \\
\hline 250 IAN & 5 & 0 & 89.427 & 10.813 & 4.836 & \\
\hline 400 IAN & 5 & 0 & 85.529 & 5.233 & 2.340 & \\
\hline 500 IAN & 5 & 0 & 84.905 & 7.103 & 3.176 & \\
\hline 600 IAN & 5 & 0 & 75.314 & 4.610 & 2.062 & \\
\hline 800 IAN & 5 & 0 & 73.314 & 3.998 & 1.788 & \\
\hline 1000 IAN & 5 & 0 & 60.759 & 6.692 & 2.993 & \\
\hline 1500 IAN & 5 & 0 & 55.306 & 6.387 & 2.856 & \\
\hline 1600 IAN & 5 & 0 & 54.652 & 3.937 & 1.761 & \\
\hline 2000 IAN & 5 & 0 & 41.919 & 5.755 & 2.574 & \\
\hline 2400 IAN & 5 & 0 & 40.621 & 2.903 & 1.298 & \\
\hline 2500 IAN & 5 & 0 & 37.108 & 6.007 & 2.686 & \\
\hline 3000 IAN & 5 & 0 & 32.876 & 4.591 & 2.053 & \\
\hline \multicolumn{2}{|c|}{ Source of Variation } & DF & SS & MS & $\mathbf{F}$ & $\mathbf{P}$ \\
\hline \multicolumn{2}{|c|}{ Between Groups } & 18 & 41021.732 & 2278.985 & 59.679 & $<0.001$ \\
\hline \multicolumn{2}{|l|}{ Residual } & 76 & 2902.250 & 38.187 & & \\
\hline \multicolumn{2}{|l|}{ Total } & 94 & 43923.982 & & & \\
\hline
\end{tabular}

The differences in the mean values among the treatment groups are greater than would be expected by chance; there is a statistically significant difference $(\mathrm{P}=<0.001)$. Power of performed test with alpha $=0.050: 1.000$

Multiple Comparisons versus Control Group (Holm-Sidak method):

Overall significance level $=0.05$

Comparisons for factor:

$\begin{array}{lcccc}\text { Comparison } & \text { Diff of Means } & \mathbf{t} & \mathbf{P} & \mathbf{P}<\mathbf{0 . 0 5 0} \\ \text { 0 IAN vs. 3000 IAN } & 67.124 & 17.175 & <0.001 & \text { Yes } \\ \text { 0 IAN vs. 2500 IAN } & 62.892 & 16.092 & <0.001 & \text { Yes } \\ \text { 0 IAN vs. 2400 IAN } & 59.379 & 15.193 & <0.001 & \text { Yes } \\ \text { 0 IAN vs. 2000 IAN } & 58.081 & 14.861 & <0.001 & \text { Yes } \\ \text { 0 IAN vs. 1600 IAN } & 45.348 & 11.603 & <0.001 & \text { Yes } \\ \text { 0 IAN vs. 1500 IAN } & 44.694 & 11.436 & <0.001 & \text { Yes } \\ \text { 0 IAN vs. 1000 IAN } & 39.241 & 10.040 & <0.001 & \text { Yes } \\ \text { 0 IAN vs. 800 IAN } & 26.686 & 6.828 & <0.001 & \text { Yes } \\ \text { 0 IAN vs. 600 IAN } & 24.686 & 6.316 & <0.001 & \text { Yes } \\ \text { 0 IAN vs. 20 IAN } & 18.368 & 4.700 & <0.001 & \text { Yes } \\ \text { 0 IAN vs. 80 IAN } & 17.074 & 4.369 & <0.001 & \text { Yes } \\ \text { 0 IAN vs. 200 IAN } & 16.345 & 4.182 & <0.001 & \text { Yes } \\ \text { 0 IAN vs. 500 IAN } & 15.095 & 3.862 & 0.001 & \text { Yes } \\ \text { 0 IAN vs. 400 IAN } & 14.471 & 3.703 & 0.002 & \text { Yes } \\ \text { 0 IAN vs. 60 IAN } & 11.923 & 3.051 & 0.013 & \text { Yes } \\ \text { 0 IAN vs. 250 IAN } & 10.573 & 2.705 & 0.025 & \text { Yes } \\ \text { 0 IAN vs. 40 IAN } & 10.534 & 2.695 & 0.017 & \text { Yes } \\ \text { 0 IAN vs. 8 IAN } & 5.360 & 1.372 & 0.174 & \text { No }\end{array}$


Table S18. One Way Analysis of Variance: Bromoacetonitrile (BAN) thiol reactivity response as the percent of the negative control.

$\begin{array}{lcccrcr}\text { Group Name } & \text { N } & \text { Missing } & \text { Mean } & \text { Std Dev } & \text { SEM } & \\ \text { 0 BAN } & 6 & 0 & 99.997 & 4.971 & 2.029 & \\ \text { 8 BAN } & 2 & 0 & 95.643 & 1.776 & 1.256 & \\ \text { 20 BAN } & 2 & 0 & 90.184 & 2.104 & 1.488 & \\ \text { 40 BAN } & 2 & 0 & 89.884 & 2.883 & 2.039 & \\ \text { 60 BAN } & 2 & 0 & 90.217 & 0.608 & 0.430 & \\ \text { 80 BAN } & 2 & 0 & 86.169 & 5.746 & 4.063 & \\ \text { 200 BAN } & 2 & 0 & 87.246 & 5.438 & 3.845 & \\ \text { 250 BAN } & 3 & 0 & 88.225 & 10.845 & 6.261 & \\ \text { 400 BAN } & 2 & 0 & 80.894 & 4.024 & 2.845 & \\ \text { 500 BAN } & 4 & 0 & 82.856 & 5.575 & 2.787 & \\ \text { 600 BAN } & 2 & 0 & 73.367 & 1.004 & 0.710 & \\ \text { 800 BAN } & 2 & 0 & 72.242 & 1.448 & 1.024 & \\ \text { 1000 BAN } & 4 & 0 & 61.187 & 7.089 & 3.544 & \\ \text { 1500 BAN } & 3 & 0 & 49.959 & 5.613 & 3.241 & \\ \text { 1600 BAN } & 2 & 0 & 47.309 & 0.717 & 0.507 & \\ \text { 2000 BAN } & 4 & 0 & 40.048 & 2.345 & 1.173 & \\ \text { 2400 BAN } & 2 & 0 & 28.826 & 3.395 & 2.401 & \\ \text { 2500 BAN } & 3 & 0 & 39.303 & 3.419 & 1.974 & \\ \text { 3000 BAN } & 4 & 0 & 27.345 & 4.842 & 2.421 & \\ & & & & & & \\ \text { Source of Variation } & \text { DF } & \text { SS } & \text { MS } & \text { F } & \text { P } \\ \text { Between Groups } & 18 & 31768.426 & 1764.913 & 67.714 & <0.001 \\ \text { Residual } & & 34 & 886.188 & 26.064 & & \\ \text { Total } & & 52 & 32654.614 & & & \end{array}$

The differences in the mean values among the treatment groups are greater than would be expected by chance; there is a statistically significant difference $(\mathrm{P}=<0.001)$. Power of performed test with alpha $=0.050: 1.000$

Multiple Comparisons versus Control Group (Holm-Sidak method):

Overall significance level $=0.05$

Comparisons for factor:

$\begin{array}{lcccc}\text { Comparison } & \text { Diff of Means } & \mathbf{t} & \mathbf{P} & \mathbf{P}<\mathbf{0 . 0 5 0} \\ \text { 0 BAN vs. 3000 BAN } & 72.652 & 22.046 & <0.001 & \text { Yes } \\ \text { 0 BAN vs. 2000 BAN } & 59.950 & 18.191 & <0.001 & \text { Yes } \\ \text { 0 BAN vs. 2400 BAN } & 71.171 & 17.074 & <0.001 & \text { Yes } \\ \text { 0 BAN vs. 2500 BAN } & 60.695 & 16.813 & <0.001 & \text { Yes } \\ \text { 0 BAN vs. 1500 BAN } & 50.038 & 13.861 & <0.001 & \text { Yes } \\ \text { 0 BAN vs. 1600 BAN } & 52.688 & 12.640 & <0.001 & \text { Yes } \\ \text { 0 BAN vs. 1000 BAN } & 38.810 & 11.777 & <0.001 & \text { Yes } \\ \text { 0 BAN vs. 800 BAN } & 27.756 & 6.658 & <0.001 & \text { Yes } \\ \text { 0 BAN vs. 600 BAN } & 26.630 & 6.388 & <0.001 & \text { Yes } \\ \text { 0 BAN vs. 500 BAN } & 17.141 & 5.201 & <0.001 & \text { Yes } \\ \text { 0 BAN vs. 400 BAN } & 19.103 & 4.583 & <0.001 & \text { Yes } \\ \text { 0 BAN vs. 80 BAN } & 13.828 & 3.317 & 0.015 & \text { Yes } \\ \text { 0 BAN vs. 250 BAN } & 11.772 & 3.261 & 0.015 & \text { Yes } \\ \text { 0 BAN vs. 200 BAN } & 12.751 & 3.059 & 0.021 & \text { Yes } \\ \text { 0 BAN vs. 40 BAN } & 10.113 & 2.426 & 0.080 & \text { No } \\ \text { 0 BAN vs. 20 BAN } & 9.814 & 2.354 & 0.072 & \text { No } \\ \text { 0 BAN vs. 60 BAN } & 9.780 & 2.346 & 0.049 & \text { Yes } \\ \text { 0 BAN vs. 8 BAN } & 4.355 & 1.045 & 0.304 & \text { No }\end{array}$


Table S19. One Way Analysis of Variance: Chloroacetonitrile (CAN) thiol reactivity response as the percent of the negative control.

$\begin{array}{lccrcrl}\text { Group Name } & \text { N } & \text { Missing } & \text { Mean } & \text { Std Dev } & \text { SEM } & \\ \text { 0 CAN } & 8 & 0 & 99.984 & 2.240 & 0.792 & \\ \text { 8 CAN } & 2 & 0 & 98.432 & 7.681 & 5.431 & \\ \text { 20 CAN } & 2 & 0 & 95.940 & 0.529 & 0.374 & \\ \text { 40 CAN } & 2 & 0 & 92.835 & 1.087 & 0.768 & \\ \text { 60 CAN } & 2 & 0 & 90.987 & 7.137 & 5.047 & \\ \text { 80 CAN } & 2 & 0 & 85.841 & 0.374 & 0.265 & \\ \text { 200 CAN } & 2 & 0 & 100.156 & 2.188 & 1.547 & \\ \text { 400 CAN } & 2 & 0 & 93.359 & 5.294 & 3.744 & \\ \text { 600 CAN } & 2 & 0 & 90.099 & 1.050 & 0.742 & \\ \text { 800 CAN } & 2 & 0 & 86.153 & 2.886 & 2.040 & \\ \text { 1600 CAN } & 2 & 0 & 107.954 & 0.000 & 0.000 & \\ \text { 2000 CAN } & 3 & 0 & 98.094 & 1.896 & 1.095 & \\ \text { 2400 CAN } & 2 & 0 & 97.362 & 0.000 & 0.000 & \\ \text { 3000 CAN } & 3 & 0 & 95.029 & 1.138 & 0.657 & \\ \text { 4000 CAN } & 3 & 0 & 91.461 & 3.579 & 2.066 & \\ \text { 5000 CAN } & 3 & 0 & 93.095 & 3.739 & 2.159 & \\ \text { 6000 CAN } & 3 & 0 & 91.853 & 3.864 & 2.231 & \\ \text { 7000 CAN } & 3 & 0 & 90.364 & 2.971 & 1.715 & \\ \text { 8000 CAN } & 6 & 0 & 95.747 & 6.696 & 2.734 & \\ \text { 10000 CAN } & 3 & 0 & 92.945 & 3.402 & 1.964 & \\ \text { 12000 CAN } & 3 & 0 & 94.295 & 3.245 & 1.874 & \\ \text { 14000 CAN } & 3 & 0 & 88.672 & 8.823 & 5.094 & \\ \text { 16000 CAN } & 3 & 0 & 97.471 & 5.899 & 3.406 & \\ \text { 18000 CAN } & 3 & 0 & 99.639 & 8.949 & 5.167 & \\ & & & & & & \\ \text { Source of Variation } & \text { DF } & \text { SS } & \text { MS } & \text { F } & \text { P } \\ \text { Between Groups } & 23 & 1434.989 & 62.391 & 2.944 & <0.001 \\ \text { Residual } & & 45 & 953.614 & 21.191 & & \\ \text { Total } & & 68 & 2388.602 & & & \end{array}$

The differences in the mean values among the treatment groups are greater than would be expected by chance; there is a statistically significant difference $(\mathrm{P}=<0.001)$.

NOTE No concentration response observed. Interpretation, no significant effect on NAC thiol reactivity observed.

Power of performed test with alpha $=0.050: 0.947$

Multiple Comparisons versus Control Group (Holm-Sidak method):

Overall significance level $=0.05$

Comparisons for factor:

$\begin{array}{lcccc}\text { Comparison } & \text { Diff of Means } & \mathbf{t} & \mathbf{P} & \mathbf{P}<\mathbf{0 . 0 5 0} \\ \text { 0 CAN vs. 80 CAN } & 14.142 & 3.886 & 0.008 & \text { Yes } \\ \text { 0 CAN vs. 800 CAN } & 13.831 & 3.800 & 0.009 & \text { Yes } \\ \text { 0 CAN vs. 14000 CAN } & 11.311 & 3.630 & 0.015 & \text { Yes } \\ \text { 0 CAN vs. 7000 CAN } & 9.620 & 3.087 & 0.067 & \text { No } \\ \text { 0 CAN vs. 4000 CAN } & 8.523 & 2.735 & 0.156 & \text { No } \\ \text { 0 CAN vs. 600 CAN } & 9.885 & 2.716 & 0.155 & \text { No } \\ \text { 0 CAN vs. 6000 CAN } & 8.131 & 2.609 & 0.190 & \text { No }\end{array}$




$\begin{array}{lllll}\text { 0 CAN vs. 60 CAN } & 8.997 & 2.472 & 0.243 & \text { No } \\ \text { 0 CAN vs. 10000 CAN } & 7.039 & 2.258 & 0.355 & \text { No } \\ \text { 0 CAN vs. 5000 CAN } & 6.888 & 2.210 & 0.368 & \text { No } \\ \text { 0 CAN vs. 1600 CAN } & 7.971 & 2.190 & 0.360 & \text { No } \\ \text { 0 CAN vs. 40 CAN } & 7.149 & 1.964 & 0.497 & \text { No } \\ \text { 0 CAN vs. 12000 CAN } & 5.688 & 1.825 & 0.574 & \text { No } \\ \text { 0 CAN vs. 400 CAN } & 6.624 & 1.820 & 0.543 & \text { No } \\ \text { 0 CAN vs. 8000 CAN } & 4.237 & 1.704 & 0.594 & \text { No } \\ \text { 0 CAN vs. 3000 CAN } & 4.954 & 1.590 & 0.637 & \text { No } \\ \text { 0 CAN vs. 20 CAN } & 4.044 & 1.111 & 0.892 & \text { No } \\ \text { 0 CAN vs. 16000 CAN } & 2.513 & 0.806 & 0.964 & \text { No } \\ \text { 0 CAN vs. 2400 CAN } & 2.621 & 0.720 & 0.960 & \text { No } \\ \text { 0 CAN vs. 2000 CAN } & 1.890 & 0.606 & 0.958 & \text { No } \\ \text { 0 CAN vs. 8 CAN } & 1.552 & 0.426 & 0.965 & \text { No } \\ \text { 0 CAN vs. 18000 CAN } & 0.345 & 0.111 & 0.992 & \text { No } \\ \text { 0 CAN vs. 200 CAN } & 0.172 & 0.0473 & 0.962 & \text { No }\end{array}$

Table S20. One Way Analysis of Variance: Bromochloroacetonitrile (BCAN) thiol reactivity response as the percent of the negative control.

$\begin{array}{lcccccc}\text { Group Name } & \text { N } & \text { Missing } & \text { Mean } & \text { Std Dev } & \text { SEM } & \\ \text { 0 BCAN } & 4 & 0 & 99.971 & 2.548 & 1.274 & \\ \text { 100 BCAN } & 3 & 0 & 93.972 & 0.298 & 0.172 & \\ \text { 200 BCAN } & 3 & 0 & 87.731 & 1.383 & 0.798 & \\ \text { 400 BCAN } & 3 & 0 & 74.443 & 1.368 & 0.790 & \\ \text { 600 BCAN } & 3 & 0 & 65.519 & 2.260 & 1.305 & \\ \text { 800 BCAN } & 3 & 0 & 54.553 & 1.575 & 0.909 & \\ \text { 900 BCAN } & 3 & 0 & 49.780 & 1.559 & 0.900 & \\ \text { 1000 BCAN } & 3 & 0 & 47.750 & 1.782 & 1.029 & \\ & & & & & & \mathbf{F} \\ \text { Source of Variation } & \text { DF } & \text { SS } & \text { MS } & \text { P } \\ \text { Between Groups } & 7 & 9604.957 & 1372.137 & 435.145 & <0.001 \\ \text { Residual } & 17 & 53.606 & 3.153 & & \\ \text { Total } & 24 & 9658.562 & & & \end{array}$

The differences in the mean values among the treatment groups are greater than would be expected by chance; there is a statistically significant difference $(\mathrm{P}=<0.001)$.

Power of performed test with alpha $=0.050: 1.000$

Multiple Comparisons versus Control Group (Holm-Sidak method):

Overall significance level $=0.05$

Comparisons for factor:

Comparison

0 BCAN vs. 1000 BCAN

0 BCAN vs. 900 BCAN

0 BCAN vs. 800 BCAN

0 BCAN vs. 600 BCAN

0 BCAN vs. 400 BCAN

0 BCAN vs. 200 BCAN

0 BCAN vs. 100 BCAN

$\begin{array}{cccc}\text { Diff of Means } & \mathbf{t} & \mathbf{P} & \mathbf{P}<\mathbf{0 . 0 5 0} \\ 52.222 & 38.504 & <0.001 & \text { Yes } \\ 50.192 & 37.008 & <0.001 & \text { Yes } \\ 45.418 & 33.488 & <0.001 & \text { Yes } \\ 34.453 & 25.403 & <0.001 & \text { Yes } \\ 25.528 & 18.823 & <0.001 & \text { Yes } \\ 12.241 & 9.025 & <0.001 & \text { Yes } \\ 6.000 & 4.424 & <0.001 & \text { Yes }\end{array}$


Table S21. One Way Analysis of Variance: Dibromoacetonitrile (DBAN) thiol reactivity response as the percent of the negative control.

$\begin{array}{lcccccc}\text { Group Name } & \text { N } & \text { Missing } & \text { Mean } & \text { Std Dev } & \text { SEM } & \\ \text { 0 DBAN } & 4 & 0 & 100.040 & 2.949 & 1.475 & \\ \text { 100 DBAN } & 3 & 0 & 85.378 & 5.280 & 3.048 & \\ \text { 200 DBAN } & 3 & 0 & 70.173 & 1.418 & 0.819 & \\ \text { 400 DBAN } & 3 & 0 & 48.588 & 1.382 & 0.798 & \\ \text { 600 DBAN } & 3 & 0 & 38.819 & 0.293 & 0.169 & \\ \text { 800 DBAN } & 3 & 0 & 29.912 & 1.548 & 0.894 & \\ \text { 900 DBAN } & 3 & 0 & 25.677 & 1.000 & 0.578 & \\ \text { 1000 DBAN } & 3 & 0 & 21.082 & 0.818 & 0.472 & \\ & & & & & & \mathbf{F} \\ \text { Source of Variation } & \text { DF } & \text { SS } & \text { MS } & \mathbf{P} \\ \text { Between Groups } & 7 & 20391.347 & 2913.050 & 505.400 & <0.001 \\ \text { Residual } & 17 & 97.986 & 5.764 & & \\ \text { Total } & 24 & 20489.333 & & & \end{array}$

The differences in the mean values among the treatment groups are greater than would be expected by chance; there is a statistically significant difference $(\mathrm{P}=<0.001)$. Power of performed test with alpha $=0.050: 1.000$

Multiple Comparisons versus Control Group (Holm-Sidak method):

Overall significance level $=0.05$

Comparisons for factor:

\section{Comparison}

0 DBAN vs. 1000 DBAN

0 DBAN vs. 900 DBAN

0 DBAN vs. 800 DBAN

0 DBAN vs. 600 DBAN

0 DBAN vs. 400 DBAN

0 DBAN vs. 200 DBAN

0 DBAN vs. 100 DBAN

Diff of Means
78.959
74.363
70.129
61.222
51.452
29.868
14.662

$\begin{array}{cc}\quad \mathbf{P} & \mathbf{P}<\mathbf{0 . 0 5 0} \\ <0.001 & \text { Yes } \\ <0.001 & \text { Yes } \\ <0.001 & \text { Yes } \\ <0.001 & \text { Yes } \\ <0.001 & \text { Yes } \\ <0.001 & \text { Yes } \\ <0.001 & \text { Yes }\end{array}$


Table S22. One Way Analysis of Variance: Dichloroacetonitrile (DCAN) thiol reactivity response as the percent of the negative control.

\begin{tabular}{lccrrrr} 
Group Name & N & Missing & \multicolumn{1}{c}{ Mean } & Std Dev & SEM & \\
0 DCAN & 9 & 0 & 100.024 & 10.540 & 3.513 & \\
2000 DCAN & 3 & 0 & 99.751 & 7.770 & 4.486 & \\
3000 DCAN & 3 & 0 & 99.700 & 3.220 & 1.859 & \\
4000 DCAN & 3 & 0 & 96.311 & 6.572 & 3.794 & \\
5000 DCAN & 3 & 0 & 97.769 & 2.245 & 1.296 & \\
6000 DCAN & 3 & 0 & 100.286 & 3.357 & 1.938 & \\
7000 DCAN & 3 & 0 & 100.949 & 5.423 & 3.131 & \\
8000 DCAN & 6 & 0 & 101.971 & 2.518 & 1.028 & \\
10000 DCAN & 3 & 0 & 98.737 & 3.435 & 1.983 & \\
12000 DCAN & 3 & 0 & 105.525 & 2.006 & 1.158 & \\
14000 DCAN & 3 & 0 & 106.053 & 4.293 & 2.479 & \\
16000 DCAN & 4 & 0 & 110.178 & 11.588 & 5.794 & \\
18000 DCAN & 4 & 0 & 105.787 & 13.892 & 6.946 & \\
& & & & & & \\
Source of Variation & DF & SS & MS & F & P \\
Between Groups & 12 & 670.924 & 55.910 & 0.903 & 0.552 \\
Residual & & 37 & 2290.002 & 61.892 & & \\
Total & 49 & 2960.926 & & &
\end{tabular}

The differences in the mean values among the treatment groups are not great enough to exclude the possibility that the difference is due to random sampling variability; there is not a statistically significant difference $(\mathrm{P}=0.552)$. 
Table S23. One Way Analysis of Variance: Tribromoacetonitrile (TBAN) thiol reactivity response as the percent of the negative control.

\begin{tabular}{|c|c|c|c|c|c|c|}
\hline Group Name & $\mathbf{N}$ & Missing & Mean & Std Dev & \multicolumn{2}{|l|}{ SEM } \\
\hline 0 TBAN & 6 & 0 & 100.018 & 2.250 & \multicolumn{2}{|l|}{0.918} \\
\hline 12.5 TBAN & 3 & 0 & 103.624 & 1.432 & \multicolumn{2}{|l|}{0.827} \\
\hline 25 TBAN & 6 & 0 & 102.615 & 2.502 & \multicolumn{2}{|l|}{1.021} \\
\hline 50 TBAN & 6 & 0 & 97.162 & 3.320 & \multicolumn{2}{|l|}{1.355} \\
\hline 100 TBAN & 6 & 0 & 79.562 & 4.924 & \multicolumn{2}{|l|}{2.010} \\
\hline 200 TBAN & 6 & 0 & 62.387 & 5.214 & \multicolumn{2}{|l|}{2.129} \\
\hline 300 TBAN & 3 & 0 & 44.157 & 2.911 & \multicolumn{2}{|l|}{1.680} \\
\hline 400 TBAN & 6 & 0 & 29.352 & 6.130 & \multicolumn{2}{|l|}{2.502} \\
\hline 800 TBAN & 6 & 0 & 1.121 & 4.588 & \multicolumn{2}{|l|}{1.873} \\
\hline \multicolumn{2}{|c|}{ Source of Variation } & DF & SS & MS & \multirow{4}{*}{$\begin{array}{c}\mathbf{F} \\
433.527\end{array}$} & \multirow{4}{*}{$\begin{array}{c}\mathbf{P} \\
<0.001\end{array}$} \\
\hline \multicolumn{2}{|c|}{ Between Groups } & 8 & 60741.593 & 7592.699 & & \\
\hline Residual & & 39 & 683.038 & 17.514 & & \\
\hline Total & & 47 & 61424.631 & & & \\
\hline
\end{tabular}

The differences in the mean values among the treatment groups are greater than would be expected by chance; there is a statistically significant difference $(\mathrm{P}=<0.001)$.

Power of performed test with alpha $=0.050: 1.000$

Multiple Comparisons versus Control Group (Holm-Sidak method):

Overall significance level $=0.05$

Comparisons for factor:

$\begin{array}{lcrcc}\text { Comparison } & \text { Diff of Means } & \mathbf{t} & \mathbf{P} & \mathbf{P}<\mathbf{0 . 0 5 0} \\ \text { 0 TBAN vs. 800 TBAN } & 98.897 & 40.931 & <0.001 & \text { Yes } \\ \text { 0 TBAN vs. 400 TBAN } & 70.666 & 29.247 & <0.001 & \text { Yes } \\ \text { 0 TBAN vs. 300 TBAN } & 55.860 & 18.877 & <0.001 & \text { Yes } \\ \text { 0 TBAN vs. 200 TBAN } & 37.631 & 15.574 & <0.001 & \text { Yes } \\ \text { 0 TBAN vs. 100 TBAN } & 20.456 & 8.466 & <0.001 & \text { Yes } \\ \text { 0 TBAN vs. 12.5 TBAN } & 3.606 & 1.219 & 0.544 & \text { No } \\ \text { 0 TBAN vs. 50 TBAN } & 2.856 & 1.182 & 0.429 & \text { No } \\ \text { 0 TBAN vs. 25 TBAN } & 2.597 & 1.075 & 0.289 & \text { No }\end{array}$


Table S24. One Way Analysis of Variance: Trichloroacetonitrile (TCAN) thiol reactivity response as the percent of the negative control.

\begin{tabular}{|c|c|c|c|c|c|c|}
\hline Group Name & $\mathbf{N}$ & Missing & Mean & Std Dev & SEM & \\
\hline 0 TCAN & 3 & 0 & 99.944 & 3.038 & 1.754 & \\
\hline 1000 TCAN & 3 & 0 & 101.627 & 8.160 & 4.711 & \\
\hline 2000 TCAN & 3 & 0 & 102.675 & 7.949 & 4.589 & \\
\hline 4000 TCAN & 3 & 0 & 103.806 & 12.212 & 7.051 & \\
\hline 6000 TCAN & 3 & 0 & 103.325 & 6.008 & 3.469 & \\
\hline 8000 TCAN & 3 & 0 & 107.048 & 7.076 & 4.086 & \\
\hline 10000 TCAN & 3 & 0 & 103.071 & 1.775 & 1.025 & \\
\hline 12000 TCAN & 3 & 0 & 97.290 & 5.395 & 3.115 & \\
\hline 14000 TCAN & 3 & 0 & 94.639 & 4.244 & 2.450 & \\
\hline 16000 TCAN & 3 & 0 & 88.948 & 2.162 & 1.248 & \\
\hline 18000 TCAN & 3 & 0 & 84.348 & 0.706 & 0.408 & \\
\hline \multicolumn{2}{|c|}{ Source of Variation } & DF & SS & MS & $\mathbf{F}$ & $\mathbf{P}$ \\
\hline \multicolumn{2}{|c|}{ Between Groups } & 10 & 1444.913 & 144.491 & 3.698 & 0.005 \\
\hline \multicolumn{2}{|c|}{ Residual } & 22 & 859.490 & 39.068 & & \\
\hline \multicolumn{2}{|l|}{ Total } & 32 & 2304.403 & & & \\
\hline
\end{tabular}

The differences in the mean values among the treatment groups are greater than would be expected by chance; there is a statistically significant difference $(\mathrm{P}=0.005)$.

Power of performed test with alpha $=0.050: 0.860$

Multiple Comparisons versus Control Group (Holm-Sidak method):

Overall significance level $=0.05$

Comparisons for factor:

Comparison

0 TCAN vs. 18000 TCAN

0 TCAN vs. 16000 TCAN

0 TCAN vs. 8000 TCAN

0 TCAN vs. 14000 TCAN

0 TCAN vs. 4000 TCAN

0 TCAN vs. 6000 TCAN

0 TCAN vs. 10000 TCAN

0 TCAN vs. 2000 TCAN

0 TCAN vs. 12000 TCAN

0 TCAN vs. 1000 TCAN

$\begin{array}{cccc}\text { Diff of Means } & \mathbf{t} & \mathbf{P} & \mathbf{P}<\mathbf{0 . 0 5 0} \\ 15.597 & 3.056 & 0.050 & \text { Yes } \\ 10.996 & 2.155 & 0.323 & \text { No } \\ 7.103 & 1.392 & 0.791 & \text { No } \\ 5.306 & 1.040 & 0.925 & \text { No } \\ 3.861 & 0.757 & 0.974 & \text { No } \\ 3.381 & 0.662 & 0.973 & \text { No } \\ 3.127 & 0.613 & 0.958 & \text { No } \\ 2.730 & 0.535 & 0.935 & \text { No } \\ 2.655 & 0.520 & 0.846 & \text { No } \\ 1.683 & 0.330 & 0.745 & \text { No }\end{array}$


Table S25. One Way Analysis of Variance: Bromodichloroacetonitrile (BDCAN) thiol reactivity response as the percent of the negative control.

$\begin{array}{lrcrccc}\text { Group Name } & \text { N } & \text { Missing } & \text { Mean } & \text { Std Dev } & \text { SEM } & \\ \text { BDCAN 0 } & 14 & 0 & 99.989 & 6.243 & 1.668 & \\ \text { 25 BDCAN } & 3 & 0 & 101.056 & 4.277 & 2.469 & \\ \text { 50 BDCAN } & 3 & 0 & 105.776 & 1.052 & 0.607 & \\ \text { 100 BDCAN } & 3 & 0 & 91.096 & 2.062 & 1.190 & \\ \text { 150 BDCAN } & 3 & 0 & 81.060 & 2.504 & 1.446 & \\ \text { 200 BDCAN } & 3 & 0 & 70.891 & 4.122 & 2.380 & \\ \text { 250 BDCAN } & 3 & 0 & 60.022 & 2.913 & 1.682 & \\ \text { 300 BDCAN } & 3 & 0 & 48.350 & 2.309 & 1.333 & \\ \text { 350 BDCAN } & 3 & 0 & 40.242 & 2.941 & 1.698 & \\ \text { 400 BDCAN } & 3 & 0 & 30.183 & 2.925 & 1.689 & \\ \text { 450 BDCAN } & 3 & 0 & 25.416 & 2.218 & 1.280 & \\ \text { 500 BDCAN } & 3 & 0 & 25.409 & 4.644 & 2.681 & \\ \text { 750 BDCAN } & 3 & 0 & -0.266 & 2.882 & 1.664 & \\ & & & & & & \text { P } \\ \text { Source of Variation } & \text { DF } & \text { SS } & \text { MS } & & \text { F } \\ \text { Between Groups } & 12 & 56808.208 & 4734.017 & 239.250 & <0.001 \\ \text { Residual } & 37 & 732.117 & 19.787 & & \\ \text { Total } & 49 & 57540.325 & & & \end{array}$

The differences in the mean values among the treatment groups are greater than would be expected by chance; there is a statistically significant difference $(\mathrm{P}=<0.001)$.

Power of performed test with alpha $=0.050: 1.000$

Multiple Comparisons versus Control Group (Holm-Sidak method):

Overall significance level $=0.05$

Comparisons for factor:

$\begin{array}{lcccc}\text { Comparison } & \text { Diff of Means } & \mathbf{t} & \mathbf{P} & \mathbf{P}<\mathbf{0 . 0 5 0} \\ \text { BDCAN 0 vs. 750 BDCAN } & 100.255 & 35.426 & <0.001 & \text { Yes } \\ \text { BDCAN 0 vs. 500 BDCAN } & 74.580 & 26.353 & <0.001 & \text { Yes } \\ \text { BDCAN 0 vs. 450 BDCAN } & 74.573 & 26.351 & <0.001 & \text { Yes } \\ \text { BDCAN 0 vs. 400 BDCAN } & 69.806 & 24.666 & <0.001 & \text { Yes } \\ \text { BDCAN 0 vs. 350 BDCAN } & 59.747 & 21.112 & <0.001 & \text { Yes } \\ \text { BDCAN 0 vs. 300 BDCAN } & 51.639 & 18.247 & <0.001 & \text { Yes } \\ \text { BDCAN 0 vs. 250 BDCAN } & 39.967 & 14.123 & <0.001 & \text { Yes } \\ \text { BDCAN 0 vs. 200 BDCAN } & 29.098 & 10.282 & <0.001 & \text { Yes } \\ \text { BDCAN 0 vs. 150 BDCAN } & 18.929 & 6.689 & <0.001 & \text { Yes } \\ \text { BDCAN 0 vs. 100 BDCAN } & 8.893 & 3.142 & 0.010 & \text { Yes } \\ \text { BDCAN 0 vs. 50 BDCAN } & 5.787 & 2.045 & 0.094 & \text { No } \\ \text { BDCAN 0 vs. 25 BDCAN } & 1.067 & 0.377 & 0.708 & \text { No }\end{array}$


Table S26. One Way Analysis of Variance: Chlorodibromoacetonitrile (CDBAN) thiol reactivity response as the percent of the negative control.

\begin{tabular}{|c|c|c|c|c|c|c|}
\hline Group Name & $\mathbf{N}$ & Missing & Mean & Std Dev & SEM & \\
\hline 0 CDBAN & 14 & 0 & 99.980 & 4.465 & 1.193 & \\
\hline 25 CDBAN & 4 & 0 & 100.473 & 5.246 & 2.623 & \\
\hline 50 CDBAN & 4 & 0 & 102.187 & 6.387 & 3.193 & \\
\hline 100 CDBAN & 4 & 0 & 86.473 & 1.304 & 0.652 & \\
\hline 150 CDBAN & 4 & 0 & 79.284 & 6.125 & 3.063 & \\
\hline 200 CDBAN & 4 & 0 & 69.843 & 4.396 & 2.198 & \\
\hline 250 CDBAN & 4 & 0 & 61.516 & 7.247 & 3.623 & \\
\hline 300 CDBAN & 4 & 0 & 49.923 & 4.354 & 2.177 & \\
\hline 350 CDBAN & 4 & 0 & 45.266 & 4.734 & 2.367 & \\
\hline 400 CDBAN & 4 & 0 & 36.870 & 5.150 & 2.575 & \\
\hline 450 CDBAN & 4 & 0 & 32.426 & 6.679 & 3.339 & \\
\hline 500 CDBAN & 4 & 0 & 23.777 & 3.786 & 1.893 & \\
\hline 550 CDBAN & 4 & 0 & 18.951 & 6.014 & 3.007 & \\
\hline 600 CDBAN & 4 & 0 & 14.995 & 2.339 & 1.170 & \\
\hline 650 CDBAN & 4 & 0 & 12.761 & 2.335 & 1.167 & \\
\hline 700 CDBAN & 4 & 0 & 12.632 & 6.065 & 3.032 & \\
\hline 750 CDBAN & 4 & 0 & 6.454 & 0.188 & 0.0939 & \\
\hline 800 CDBAN & 3 & 0 & 2.652 & 0.107 & 0.0618 & \\
\hline \multicolumn{2}{|c|}{ Source of Variation } & DF & SS & MS & $\mathbf{F}$ & $\mathbf{P}$ \\
\hline \multicolumn{2}{|c|}{ Between Groups } & 17 & 104161.331 & 6127.137 & 270.028 & $<0.001$ \\
\hline \multicolumn{2}{|c|}{ Residual } & 63 & 1429.517 & \multicolumn{2}{|l|}{22.691} & \\
\hline Total & & 80 & 105590.847 & & & \\
\hline
\end{tabular}

The differences in the mean values among the treatment groups are greater than would be expected by chance; there is a statistically significant difference $(\mathrm{P}=<0.001)$. Power of performed test with alpha $=0.050: 1.000$

Multiple Comparisons versus Control Group (Holm-Sidak method):

Overall significance level $=0.05$

Comparisons for factor:

$\begin{array}{lcccc}\text { Comparison } & \text { Diff of Means } & \mathbf{t} & \mathbf{P} & \mathbf{P}<\mathbf{0 . 0 5 0} \\ \text { 0 CDBAN vs. 750 CDBAN } & 93.526 & 34.631 & <0.001 & \text { Yes } \\ \text { 0 CDBAN vs. 700 CDBAN } & 87.348 & 32.344 & <0.001 & \text { Yes } \\ \text { 0 CDBAN vs. 650 CDBAN } & 87.220 & 32.296 & <0.001 & \text { Yes } \\ \text { 0 CDBAN vs. 800 CDBAN } & 97.328 & 32.115 & <0.001 & \text { Yes } \\ \text { 0 CDBAN vs. 600 CDBAN } & 84.986 & 31.469 & <0.001 & \text { Yes } \\ \text { 0 CDBAN vs. 550 CDBAN } & 81.029 & 30.004 & <0.001 & \text { Yes } \\ \text { 0 CDBAN vs. 500 CDBAN } & 76.203 & 28.217 & <0.001 & \text { Yes } \\ \text { 0 CDBAN vs. 450 CDBAN } & 67.554 & 25.014 & <0.001 & \text { Yes } \\ \text { 0 CDBAN vs. 400 CDBAN } & 63.110 & 23.369 & <0.001 & \text { Yes } \\ \text { 0 CDBAN vs. 350 CDBAN } & 54.714 & 20.260 & <0.001 & \text { Yes } \\ \text { 0 CDBAN vs. 300 CDBAN } & 50.058 & 18.535 & <0.001 & \text { Yes } \\ \text { 0 CDBAN vs. 250 CDBAN } & 38.464 & 14.243 & <0.001 & \text { Yes } \\ \text { 0 CDBAN vs. 200 CDBAN } & 30.138 & 11.159 & <0.001 & \text { Yes } \\ \text { 0 CDBAN vs. 150 CDBAN } & 20.696 & 7.663 & <0.001 & \text { Yes } \\ \text { 0 CDBAN vs. 100 CDBAN } & 13.507 & 5.002 & <0.001 & \text { Yes } \\ \text { 0 CDBAN vs. 50 CDBAN } & 2.207 & 0.817 & 0.660 & \text { No } \\ \text { 0 CDBAN vs. 25 CDBAN } & 0.493 & 0.182 & 0.856 & \text { No }\end{array}$


Table S27. One Way Analysis of Variance: HAN TRI Comparisons.

\begin{tabular}{lccllll} 
Group Name & N & Missing & Mean & Std Dev & \multicolumn{1}{c}{ SEM } & \\
IAN TRI & 6 & 0 & 0.589 & 0.0631 & 0.0258 & \\
BAN TRI & 6 & 0 & 0.666 & 0.0186 & 0.00761 & \\
DBAN TRI & 6 & 0 & 2.478 & 0.0587 & 0.0240 \\
BCAN TRI & 6 & 0 & 1.096 & 0.0326 & 0.0133 & \\
TBAN TRI & 9 & 0 & 3.820 & 0.279 & 0.0931 & \\
TCAN TRI & 6 & 0 & 0.0382 & 0.000214 & 0.0000875 & \\
BDCAN TRI & 11 & 0 & 3.309 & 0.0745 & 0.0225 & \\
CDBAN TRI & 11 & 0 & 3.190 & 0.183 & 0.0553 & \\
& & & & & & P \\
Source of Variation & DF & SS & MS & F & \\
Between Groups & & 7 & 113.463 & 16.209 & 809.632 & $<0.001$ \\
Residual & & 53 & 1.061 & 0.0200 & & \\
Total & 60 & 114.524 & & &
\end{tabular}

The differences in the mean values among the treatment groups are greater than would be expected by chance; there is a statistically significant difference $(\mathrm{P}=<0.001)$.

Power of performed test with alpha $=0.050: 1.000$

All Pairwise Multiple Comparison Procedures (Holm-Sidak method):

Overall significance level $=0.05$

Comparisons for factor:

$\begin{array}{lcccc}\text { Comparison } & \text { Diff of Means } & \mathbf{t} & \mathbf{P} & \text { P }<\mathbf{0 . 0 5 0} \\ \text { TBAN TRI vs. TCAN TRI } & 3.782 & 50.717 & <0.001 & \text { Yes } \\ \text { BDCAN TRI vs. TCAN TRI } & 3.271 & 45.553 & <0.001 & \text { Yes } \\ \text { CDBAN TRI vs. TCAN TRI } & 3.152 & 43.896 & <0.001 & \text { Yes } \\ \text { TBAN TRI vs. IAN TRI } & 3.232 & 43.338 & <0.001 & \text { Yes } \\ \text { TBAN TRI vs. BAN TRI } & 3.155 & 42.302 & <0.001 & \text { Yes } \\ \text { BDCAN TRI vs. IAN TRI } & 2.721 & 37.890 & <0.001 & \text { Yes } \\ \text { BDCAN TRI vs. BAN TRI } & 2.644 & 36.814 & <0.001 & \text { Yes } \\ \text { TBAN TRI vs. BCAN TRI } & 2.724 & 36.529 & <0.001 & \text { Yes } \\ \text { CDBAN TRI vs. IAN TRI } & 2.602 & 36.233 & <0.001 & \text { Yes } \\ \text { CDBAN TRI vs. BAN TRI } & 2.525 & 35.157 & <0.001 & \text { Yes } \\ \text { BDCAN TRI vs. BCAN TRI } & 2.213 & 30.819 & <0.001 & \text { Yes } \\ \text { DBAN TRI vs. TCAN TRI } & 2.440 & 29.863 & <0.001 & \text { Yes } \\ \text { CDBAN TRI vs. BCAN TRI } & 2.094 & 29.162 & <0.001 & \text { Yes } \\ \text { DBAN TRI vs. IAN TRI } & 1.889 & 23.126 & <0.001 & \text { Yes } \\ \text { DBAN TRI vs. BAN TRI } & 1.812 & 22.181 & <0.001 & \text { Yes } \\ \text { TBAN TRI vs. DBAN TRI } & 1.343 & 18.005 & <0.001 & \text { Yes } \\ \text { DBAN TRI vs. BCAN TRI } & 1.381 & 16.911 & <0.001 & \text { Yes } \\ \text { BCAN TRI vs. TCAN TRI } & 1.058 & 12.952 & <0.001 & \text { Yes } \\ \text { BDCAN TRI vs. DBAN TRI } & 0.832 & 11.581 & <0.001 & \text { Yes } \\ \text { CDBAN TRI vs. DBAN TRI } & 0.713 & 9.925 & <0.001 & \text { Yes } \\ \text { TBAN TRI vs. CDBAN TRI } & 0.630 & 9.906 & <0.001 & \text { Yes } \\ \text { TBAN TRI vs. BDCAN TRI } & 0.511 & 8.035 & <0.001 & \text { Yes } \\ \text { BAN TRI vs. TCAN TRI } & 0.628 & 7.682 & <0.001 & \text { Yes } \\ \text { IAN TRI vs. TCAN TRI } & 0.550 & 6.736 & <0.001 & \text { Yes } \\ \text { BCAN TRI vs. IAN TRI } & 0.508 & 6.216 & <0.001 & \text { Yes } \\ \text { BCAN TRI vs. BAN TRI } & 0.431 & 5.270 & <0.001 & \text { Yes } \\ \text { BDCAN TRI vs. CDBAN TRI } & 0.119 & 1.972 & 0.105 & \text { No } \\ \text { BAN TRI vs. IAN TRI } & 0.0773 & 0.946 & 0.349 & \text { No }\end{array}$




\section{References}

1. Chen, B.; Zhang, T.; Bond, T.; Gan, Y., Development of quantitative structure activity relationship (QSAR) model for disinfection byproduct (DBP) research: A review of methods and resources. J. Hazard. Mater. 2015, 299, 260-279.

2. Gramatica, P.; Cassani, S.; Sangion, A., Aquatic ecotoxicity of personal care products: QSAR models and ranking for prioritization and safer alternatives' design. Green Chemistry 2016, 18, (16), 4393-4406.

3. Box, G. E. P.; Hunter, W. G.; Hunter, J. S., Statistics for Experimenters: An Introduction to Design, Data Analysis, and Model Building. Wiley \& Sons Inc.: New York, NY., 1978.

4. Lovell, D. P.; Omori, T., Statistical issues in the use of the comet assay. Mutagenesis 2008, 23, (3), 171-182.

5. Efron, B., Better bootstrap confidence intervals. J. Am. Statis. Assoc. 1987, 82, (397), 171185.

6. Singh, K.; Xie, M. Bootstrap: A Statistical Method; Rutgers University: New Brunswick, NJ, 2008; p 14.

7. Plewa, M. J.; Wagner, E. D.; Richardson, S. D.; Thruston, A. D., Jr.; Woo, Y. T.; McKague, A. B., Chemical and biological characterization of newly discovered iodoacid drinking water disinfection byproducts. Environ. Sci. Technol. 2004, 38, (18), 4713-4722.

8. Li, J.; Moe, B.; Vemula, S.; Wang, W.; Li, X.-F., Emerging disinfection byproducts, halobenzoquinones: effects of isomeric structure and halogen substitution on cytotoxicity, formation of reactive oxygen species, and genotoxicity. Environ. Sci. Technol. 2016, 50, (13), 6744-6752.

9. Wagner, E. D.; Rayburn, A. L.; Anderson, D.; Plewa, M. J., Analysis of mutagens with single cell gel electrophoresis, flow cytometry, and forward mutation assays in an isolated clone of Chinese hamster ovary cells. Environ. Mol. Mutagen. 1998, 32, (4), 360-368.

10. Wagner, E. D.; Rayburn, A. L.; Anderson, D.; Plewa, M. J., Calibration of the single cell gel electrophoresis assay, flow cytometry analysis and forward mutation in Chinese hamster ovary cells. Mutagenesis 1998, 13, (1), 81-84.

11. Wagner, E. D.; Plewa, M. J., CHO cell cytotoxicity and genotoxicity analyses of disinfection by-products: an updated review. J. Environ. Sci. 2017, 58, 64-76.

12. Muellner, M. G.; Wagner, E. D.; McCalla, K.; Richardson, S. D.; Woo, Y. T.; Plewa, M. J., Haloacetonitriles vs. regulated haloacetic acids: Are nitrogen containing DBPs more toxic? Environ. Sci. Technol. 2007, 41, (2), 645-651.

13. Plewa, M. J.; Wagner, E. D., Mammalian Cell Cytotoxicity and Genotoxicity of Disinfection By-Products. Water Research Foundation: Denver, CO, 2009; p 134.

14. Tice, R. R.; Agurell, E.; Anderson, D.; Burlinson, B.; Hartmann, A.; Kobayashi, H.; Miyamae, Y.; Rojas, E.; Ryu, J. C.; Sasaki, Y. F., Single cell gel/comet assay: guidelines for in vitro and in vivo genetic toxicology testing. Environ. Mol. Mutagen. 2000, 35, (3), 206-221.

15. Rundell, M. S.; Wagner, E. D.; Plewa, M. J., The comet assay: genotoxic damage or nuclear fragmentation? Environ. Mol. Mutagen. 2003, 42, (2), 61-67.

16. Wagner, E. D.; Plewa, M. J., Microplate-based comet assay. In The Comet Assay in Toxicology, Dhawan, A.; Anderson, D., Eds. Royal Society of Chemistry: London, 2009; pp 79-97. 
17. Dong, S.; Page, M. A.; Wagner, E. D.; Plewa, M. J., Thiol reactivity analyses to predict mammalian cell cytotoxicity of water samples. Environ. Sci. Technol. 2018, 52, 8822-8829.

18. Pals, J. A.; Wagner, E. D.; Plewa, M. J.; Xia, M.; Attene-Ramos, M. S., Monohalogenated acetamide-induced cellular stress and genotoxicity are related to electrophilic softness and thiol/thiolate reactivity. J. Environ. Sci. 2017, 58, 224-230.

19. Pals, J. A.; Wagner, E. D.; Plewa, M. J., Energy of the lowest unoccupied molecular orbital, thiol reactivity, and toxicity of three monobrominated water disinfection byproducts. Environ. Sci. Technol. 2016, 50, (6), 3215-3221.

20. Townsend, D. M.; Tew, K. D.; Tapiero, H., The importance of glutathione in human disease. Biomed. Pharmacother. 2003, 57, (3-4), 145-155.

21. Meister, A.; Anderson, M. E., Glutathione. Annu. Rev. Biochem. 1983, 52, 711-760. 